\title{
Genome-scale reconstruction and system level investigation of the metabolic network of Methylobacterium extorquens AM1
}

Rémi Peyraud ${ }^{1}$, Kathrin Schneider ${ }^{1}$, Patrick Kiefer ${ }^{1}$, Stéphane Massou ${ }^{2,3,4}$, Julia A Vorholt ${ }^{1}$ and Jean-Charles Portais $2,3,4^{*}$

\begin{abstract}
Background: Methylotrophic microorganisms are playing a key role in biogeochemical processes - especially the global carbon cycle - and have gained interest for biotechnological purposes. Significant progress was made in the recent years in the biochemistry, genetics, genomics, and physiology of methylotrophic bacteria, showing that methylotrophy is much more widespread and versatile than initially assumed. Despite such progress, system-level description of the methylotrophic metabolism is currently lacking, and much remains to understand regarding the network-scale organization and properties of methylotrophy, and how the methylotrophic capacity emerges from this organization, especially in facultative organisms.

Results: In this work, we report on the integrated, system-level investigation of the metabolic network of the facultative methylotroph Methylobacterium extorquens AM1, a valuable model of methylotrophic bacteria. The genome-scale metabolic network of the bacterium was reconstructed and contains 1139 reactions and 977 metabolites. The sub-network operating upon methylotrophic growth was identified from both in silico and experimental investigations, and ${ }^{13} \mathrm{C}$-fluxomics was applied to measure the distribution of metabolic fluxes under such conditions. The core metabolism has a highly unusual topology, in which the unique enzymes that catalyse the key steps of $\mathrm{C} 1$ assimilation are tightly connected by several, large metabolic cycles (serine cycle, ethylmalonylCoA pathway, TCA cycle, anaplerotic processes). The entire set of reactions must operate as a unique process to achieve C1 assimilation, but was shown to be structurally fragile based on network analysis. This observation suggests that in nature a strong pressure of selection must exist to maintain the methylotrophic capability. Nevertheless, substantial substrate cycling could be measured within C2/C3/C4 inter-conversions, indicating that the metabolic network is highly versatile around a flexible backbone of central reactions that allows rapid switching to multi-carbon sources.

Conclusions: This work emphasizes that the metabolism of $M$. extorquens AM1 is adapted to its lifestyle not only in terms of enzymatic equipment, but also in terms of network-level structure and regulation. It suggests that the metabolism of the bacterium has evolved both structurally and functionally to an efficient but transitory utilization of methanol. Besides, this work provides a basis for metabolic engineering to convert methanol into value-added products.
\end{abstract}

\footnotetext{
* Correspondence: jean-charles.portais@insa-toulouse.fr

Université de Toulouse; INSA, UPS, INP; LISBP, 135 Avenue de Rangueil, F-

31077 Toulouse, France

Full list of author information is available at the end of the article
} 


\section{Background}

Methylotrophs are microorganisms able to grow on reduced $\mathrm{C} 1$ compounds such as methane and methanol as sole source of carbon and energy. Methylotrophy has gained increasing interest over the past decade for both basic and applied purposes, since methanol can be produced from diverse renewable sources and represents a valuable feedstock for biotechnological applications $[1,2]$. Recent progress in the biochemistry, genetics, genomics, and physiology of methylotrophic bacteria has shown that methylotrophy is a phenomenon much more widespread and versatile than initially assumed [3]. The phylogenetic distribution of methylotrophy is broad and spans over a range of phyla and genera [3]. Methylotrophic bacteria are adapted to various lifestyles and ecological niches (soil, water sediments, plant roots, phyllosphere), and are involved in a number of important biological or biogeochemical processes, in particular the global carbon cycle. Methylotrophy encompasses diverse metabolic capabilities or behaviors that were used to classify them, e.g. obligate vs facultative methyltrophy, heterotrophic vs autotrophic growth, aerobic vs anaerobic metabolism. Such genetic and biochemical diversity may explain the ecological success of methylotrophs, which can represent dominant microbial populations in specific environments, e.g. plant leaves [4].

From the biochemical point of view, methylotrophy relies on specific pathways ( $\mathrm{C} 1$ pathways) ensuring all growth requirements. Energetic requirements are enabled by dissimilation pathways, which involve a series of three basic steps: i) the oxidation of primary $\mathrm{C} 1$ substrates - methanol, methylamine - typically into the toxic intermediate formaldehyde, ii) the oxidation of the latter compound into formate, and iii) formate oxidation into carbon dioxide $\left(\mathrm{CO}_{2}\right)$. In addition, alternative dissimilation mechanism exist in which formate is not involved. The assimilation of $\mathrm{C} 1$-units can be achieved by different mechanisms starting from either formaldehyde (ribulose-monophosphate pathway, RuMP), $\mathrm{CO}_{2}$ (Calvin-Benson-Bassham cycle, $\mathrm{CBB}$ ) or methylene-tetrahydrofolate (Me-THF) $+\mathrm{CO}_{2}$ (serine cycle). Novel enzymes, biochemical mechanisms, and metabolic pathways have been discovered, resulting in a more complete description of methylotrophic pathways and their diversity. Interestingly, these findings do not modify our current view of the general organization of $\mathrm{C} 1$ pathways, but show that many more alternative enzymes or pathways than initially assumed carry out each of the basic steps of methylotrophy. Moreover, a growing number of newly discovered methylotrophs have been investigated, showing that different combinations of the various pathways can be found in nature, and leading to the concept of a modular metabolism [5].
Considerable progress was made in the understanding of the biochemistry and physiology of methylotrophy, and valuable insights were obtained regarding the organization and operation of central carbon metabolism [6-8]. However, a complete, system-level description of methylotrophy metabolism is currently lacking. The comprehensive understanding of bacterial physiology requires a detailed knowledge of the complete metabolic potential of the studied organism, but such knowledge is currently missing and no genome-scale metabolic network has been so far established for any methylotrophic bacterium. In consequence, little is currently known about the network-scale organization of methylotrophy, the specific properties of methylotrophic networks, and how the methylotrophic capacity emerges from this organization, especially in facultative representatives. Among the recently sequenced organisms, the Alphaproteobacterium Methylobacterium extorquens AM1 is a major model of methylotrophic bacteria. This facultative methylotroph is able to grow on C1- but also on multicarbon (C2-C4) compounds. Methylobacterium spp. are part of the abundant population of bacteria systematically found in the phyllosphere $[4,9]$, where they benefit from plant-derived methanol $[10,11]$. The biochemistry, genetics, and physiology of M. extorquens AM1 has been extensively investigated, and allowed the discovery of major methylotrophic pathways, including the serine cycle [12-14], the tetrahydromethanopterin $\left(\mathrm{H}_{4} \mathrm{MPT}\right)$ dependent pathway for formaldehyde oxidation $[15,16]$, and of a number of novel enzymes or enzyme functions (e.g., pyrroloquinoline quinine (PQQ)-dependent methanol dehydrogenase, formaldehyde activating enzyme, methylene- $\mathrm{H}_{4} \mathrm{MPT}$ dehydrogenases coupled to pyridine nucleotides, and formyl-methanofuran hydrolase). The central metabolic pathways were progressively unraveled, and the application of novel experimental strategies metabolic modeling, metabolic flux analysis, omics technologies - provided valuable insights into the topology and operation of central carbon metabolism $[6-8,17]$. Finally, the pathway for methanol assimilation in $M$. extorquens AM1 was recently completed with the discovery of the ethylmalonyl-CoA pathway (EMCP), an alternative to the glyoxylate cycle for the synthesis of glyoxylate, which encompasses an unusual series of 12 reactions where intermediates are all Coenzyme A (CoA) esters $[18,19]$. Finally, the genome of this bacterium was recently sequenced and annotated [20], providing inevitable information for genome-scale investigations.

In this work, we report on the integrated, system-level investigation of the metabolic network of the facultative methylotroph $M$. extorquens AM1, as a valuable model of methylotrophic bacteria. In order to obtain a comprehensive understanding of the biochemistry and physiology of 
this bacterium, we first evaluated its complete metabolic potential by compiling current biochemical knowledge and genome annotation data into a comprehensive genome-scale representation of the metabolic network. Then, we performed both in silico and experimental investigations to identify the subnetwork operating during methylotrophic growth, in order to analyze structurally and functionally the system-level organization of methylotrophy with an emphasis on the properties of the organization of central carbon metabolism.

\section{Results}

\section{Metabolic network reconstruction}

The genome-scale (GS) metabolic network of $M$. extorquens AM1 was reconstructed according to previously established guidelines [21]. The details of the process are given in material and methods and are schematically shown in Additional file 1. Briefly, the GS metabolic network was obtained by integrating relevant information collected from i) genome annotation [20], ii) published physiological, genetic and biochemical studies in M. extorquens AM1 and closely related organisms iii) biochemical information contained in databases [22-24], vi) complementary investigations (biomass quantification), and intensive refinement (Additional file 2, 3). The chemical composition of M. extorquens AM1 cell was determined experimentally or taken from available literature (Table 1 and Additional file 4) and used to define the biosynthetic needs and corresponding pathways.

The refined reconstruction was converted into a mathematical model using CellNetAnalyser [25] and the network was checked for self-consistency and curated to allow biosynthesis of all cell components from each of the 12 carbon sources established for $M$. extorquens (Additional file 5). In addition, flux balance analysis (FBA) was used to calculate the theoretical maximum growth yields for each carbon source. For methanol and succinate, the theoretical maximum growth rates could also be calculated and were in agreement with published data (Additional file 5), showing the consistency of the network with experimental observations. Last, the capability of the GS network to explain the oxidation of carbon compounds [26] was validated (Additional file 5).

Table 1 Chemical content and physiological parameters of $M$. extorquens AM1 cells growing on methanol

\begin{tabular}{|c|c|c|c|c|}
\hline Macromolecule & \multicolumn{2}{|c|}{$\%$ Cell Dry Weight $\pm \sigma$} & Data source & Organism source \\
\hline Protein & \multicolumn{2}{|l|}{$59.13 \pm 2.11$} & This study & M. extorquens AM1 \\
\hline Carbohydrate & \multicolumn{2}{|l|}{$16.43 \pm 1.09$} & This study & M. extorquens AM1 \\
\hline Rhamnose (polymer) & \multicolumn{2}{|l|}{$8.92 \pm 0.92$} & This study & M. extorquens AM1 \\
\hline Glucose (polymer) & \multicolumn{2}{|l|}{$5.62 \pm 0.52$} & This study & M. extorquens AM1 \\
\hline Trehalose & \multicolumn{2}{|l|}{$1.22 \pm 0.20$} & This study & M. extorquens AM1 \\
\hline Glucosamine (polymer) & \multicolumn{2}{|l|}{$0.09 \pm 0.67$} & This study & M. extorquens AM1 \\
\hline RNA & \multicolumn{2}{|l|}{$8.20 \pm 0.68$} & This study & M. extorquens AM1 \\
\hline Fatty acid & \multicolumn{2}{|l|}{$4.95 \pm 0.29$} & This study & M. extorquens AM1 \\
\hline DNA & \multicolumn{2}{|l|}{$3.00-$} & Neidhart et al.; GC content: Vuilleumier et al. (2009) & E. coli \\
\hline PHB & \multicolumn{2}{|l|}{$2.36 \pm 0.05$} & This study & M. extorquens AM1 \\
\hline Polyamine & \multicolumn{2}{|l|}{$0.40-$} & Neidhart et al. & E. coli \\
\hline Carotenoid & \multicolumn{2}{|l|}{$0.023-$} & Konovalova et al. (2007) & M. extorquens AM1 \\
\hline Intracellular metabolites & \multicolumn{2}{|l|}{$2.64-$} & $\begin{array}{l}\text { Kiefer et al. (2008); Guo et al. (2006); Guo et al. (2007); Vorholt } \\
\text { et al. (1998); Crowther et al. (2008) }\end{array}$ & M. extorquens AM1 \\
\hline Inorganic ions & \multicolumn{2}{|l|}{$1.01-$} & Neidhart et al. & E. coli \\
\hline Cofactors & \multicolumn{2}{|l|}{$0.22-$} & Neidhart et al. & E. coli \\
\hline SUM & \multicolumn{2}{|l|}{98.36} & & \\
\hline Physiological parameters & value $\pm \sigma$ & units & Data sources & Organism source \\
\hline Growth rate & $0.168 \pm 0.003$ & $h^{-1}$ & This study & M. extorquens AM1 \\
\hline $\begin{array}{l}\text { Specific methanol uptake } \\
\text { rate }\end{array}$ & $15.0 \pm 0.25$ & mmol.g. $g^{-1} \cdot h^{-1}$ & This study & M. extorquens AM1 \\
\hline $\begin{array}{l}\text { Specific proton production } \\
\text { rate }\end{array}$ & $0.22 \pm 0.01$ & mmol.g. $g^{-1} \cdot h^{-1}$ & This study & M. extorquens AM1 \\
\hline $\begin{array}{l}\text { Growth-associated ATP } \\
\text { maintenance }\end{array}$ & $59.81-$ & mmol.g ${ }^{-1}$ & Neidhart et al. & E. coli \\
\hline $\begin{array}{l}\text { Macromolecular } \\
\text { building costs }\end{array}$ & $26.65-$ & mmol.g ${ }^{-1}$ & This study & M. extorquens AM1 \\
\hline $\begin{array}{l}\text { Non-Growth-associated } \\
\text { ATP maintenance }\end{array}$ & $9.5-$ & mmol.g. $g^{-1} \cdot h^{-1}$ & Rokem et al. (1978) & Methylobacterium \\
\hline
\end{tabular}


The final GS network (iRP911) contained 1139 unique reactions and 977 metabolites, and was based on a gene-to-protein-to-reaction (GPR) association network that included 911 genes encoding 761 proteins (Table 2, Additional file 2, 3). The confidence in the network information was established by scoring the evidence currently available for each reaction [21]. The confidence scores ranged from 0 (lowest) to 4 (highest), with the latter being assigned to a reaction with direct evidence for both gene product function and biochemical reaction (Table 2). The average confidence score over the final network score was 2.1.

\section{Main features of the genome-scale metabolic network of M. extorquens AM1}

M. extorquens AM1 is a facultative methylotroph able to utilize a relatively narrow range of substrates. The ability to grow on $\mathrm{C} 3$ and $\mathrm{C} 4$ organic acids - e.g. lactate, pyruvate, succinate or malate - relies on the presence of common metabolic pathways, which include the tricarboxylic acid (TCA) cycle, anaplerotic pathways, gluconeogenesis, pentose-phosphate pathway (PPP), and Entner-Doudoroff (ED) pathway (Figure 1). Growth on C1 compounds relies on specific metabolic pathways that were resolved for this bacterium in the past 50 years $[5,16]$. The first step is the oxidation of primary $\mathrm{C} 1$ substrates - e.g. methanol - to formate via methanol dehydrogenase and the $\mathrm{H}_{4} \mathrm{MPT}$-dependent $\mathrm{C} 1$ pathway. Formate is a key branch-point to trigger the flow of carbon between dissimilation and assimilation. Dissimilation is achieved by oxidation of formate into $\mathrm{CO}_{2}$. The assimilation of $\mathrm{C} 1$ units requires the conversion of formate into methylene tetrahydrofolate (Me-THF), since the spontaneous condensation of formaldehyde with THF was demonstrated not to be significant [7]. In the serine cycle, Me-THF is condensed with a $\mathrm{C} 2$ compound - generated from glyoxylate - to build C3 compounds such as 2-phosphoglycerate and phosphoenolpyruvate (PEP), which are further carboxylated to form oxaloacetate (OAA) and other $\mathrm{C} 4$ intermediates. The continuous operation of the serine cycle requires the operation of the recently discovered EMCP which allows glyoxylate regeneration and involves $\mathrm{CO}_{2}$ fixation. These pathways are tightly embedded into each other and the consequences of such organization will be detailed later. The $\mathrm{C} 2$ compounds used as carbon source enter metabolism at the level of the EMCP, from which they feed the central pathways. M. extorquens possesses also the ability to oxidize 26 additional compounds [26]. These compounds include a significant number of sugars, mainly pentoses. The reconstruction data suggest that such capability is due to the occurrence of soluble sugar dehydrogenases able to oxidize a wide range of pentoses and other sugars [27]; however, no assimilation processes were identified from the genome annotation. The reconstructed network contains a potential pathway for the utilization of two sugars (glucose and gluconate) as carbon source, although $M$. extorquens is not known to grow on these compounds. The identified pathway includes the periplasmic oxidation of glucose into gluconate, which could be internalized and catabolized through the pentose phosphate pathway (PPP) or the Entner-Doudoroff (ED) pathway. The glycolytic pathways are quite well established (see below) but the relevant sugar transport systems have low annotation confidence scores. Further experimental investigations will be necessary to determine whether $M$. extorquens possesses or not the entire enzymatic repertoire for sugar utilization.

The detailed examination of the biosynthetic pathways included in the GS network indicated incomplete lipopolysaccharide (LPS) biosynthesis. The pathways for keto-deoxyoctulosonate and lipid A synthesis and

Table 2 Properties of the genome-scale (iRP911) and methylotrophic networks reconstructed for M. extorquens AM1

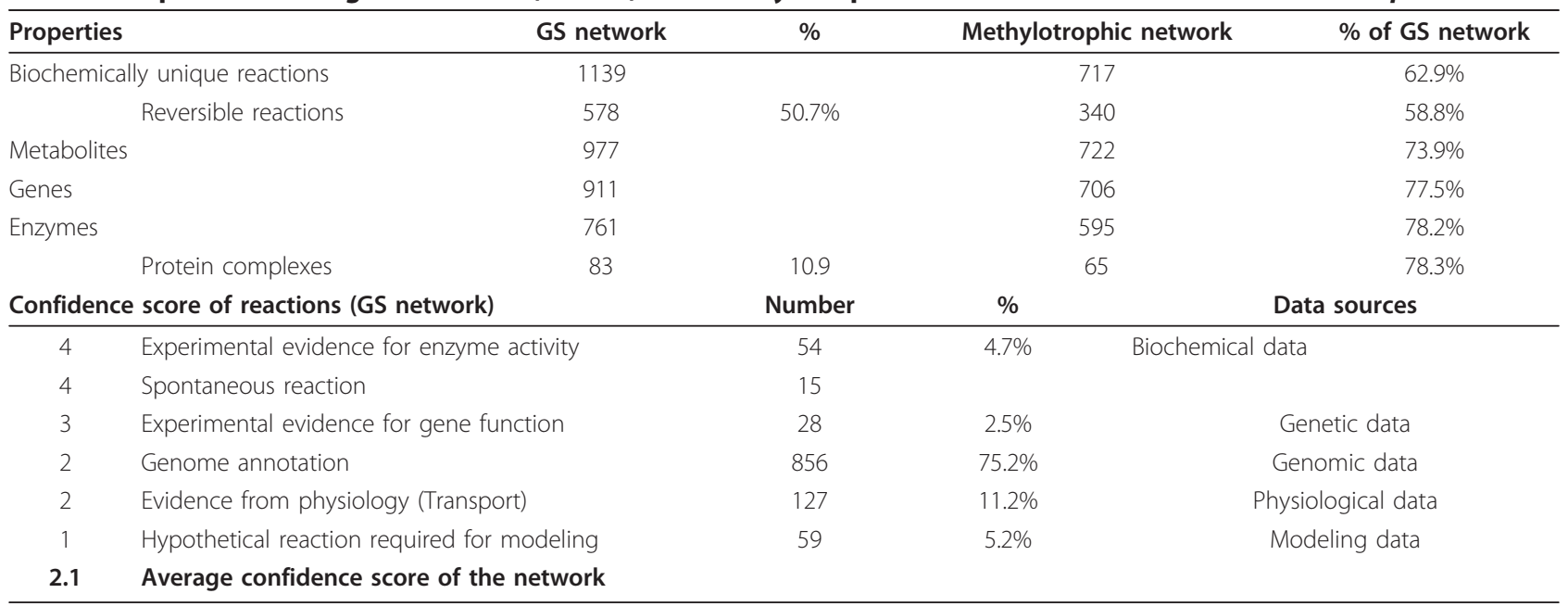


assembly were found, but the genes encoding the enzymes classically involved in heptose biosynthesis and in sugar incorporation onto the lipid A were missing. These observations suggest the occurrence of an unusual LPS structure in M. extorquens AM1. Consistently, no heptose or galactose was detected from the hydrolysis of cell material (see above) though significant contents in rhamnose $(8.9 \pm 0.9 \%)$ and glucose $(5.6 \pm 0.5 \%)$ were found. The GS network also included the biosynthetic pathways for carotenoids and bacteriochlorophyll A. The biosynthetic pathway of bacteriochlorophyll was complete in addition with the presence of the enzymes of the photosystem I. Several degradation pathways are missing in the GS network of $M$. extorquens, including the degradation of amino acids - e.g. histidine, arginine, tyrosine - and nucleotides. This is in agreement with physiological data showing that the occurrence of these compounds in the cultivation medium did not result in detectable metabolic activity.

The GS metabolic reconstruction showed that $M$. extorquens possesses a respiratory chain with alternative systems for electron inputs and outputs (Additional file 6). A great variety of potential electron donors could be identified, including a significant number of soluble periplasmic dehydrogenases transferring electrons to cytochrome c, including methanol dehydrogenase, formaldehyde dehydrogenase, and the already-mentioned soluble sugar dehydrogenase. Three terminal oxidases were also present, including two ubiquinol oxidases and one cytochrome c oxidase, suggesting that $M$. extorquens can adapt an aerobic metabolism to different oxygen levels. Besides oxygen, nitrate might represent a potential alternative electron acceptor.

\section{Organization of central metabolic pathways}

$\mathrm{C} 1$ assimilation ensures the conversion of the $\mathrm{C} 1$-unit into precursor metabolites and involves a high number of metabolic pathways like $\mathrm{C} 1$ pathways, serine cycle, EMCP, TCA cycle, gluconeogenesis, anaplerotic reactions (Figure 1). They are connected by overlapping metabolites and enzyme reactions. The most central processes are interconnected cycles (serine cycle, TCA cycle) or pathways (EMCP, anaplerotic reactions). The serine cycle shares common reactions with gluconeogenesis (enolase), with the EMCP (malyl-CoA ligase, malylCoA lyase), with the TCA cycle (malate dehydrogenase), and with amino acid metabolism (serine hydroxymethyltransferase). The EMCP shares also reactions with the polyhydroxybutyrate (PHB) biosynthesis (acetyl-CoA Cacetyltransferase, acetoacetyl-CoA reductase), the TCA cycle (succinate dehydrogenase, fumarase), and fatty acid degradation (hydroxybutyryl-CoA (HBCOA) dehydratase, hydroxybutyryl-CoA dehydrogenase). The overall picture of $M$. extorquens central metabolism that emerges from these observations is an unusual series of metabolic pathways and cycles that are tightly embedded one into each other and allow operating almost as an entity. The C3 (2PG, pyruvate and PEP) and C4 (OAA and malate) intermediates play a critical role in the overall network organization. They represent the branching-points of the three main central metabolic pathways, i.e. the serine cycle, the EMCP and the TCA cycle, and of anaplerotic processes. Hence, they can be generated by different metabolic routes [28]. Accordingly, seven different reactions allow the inter-conversion of the five compounds (Figure 1). These reactions include processes inter-converting i) C3 into C3 (enolase, PEP synthase, pyruvate kinase), ii) $\mathrm{C} 4$ into $\mathrm{C} 4$ (malate dehydrogenase), iii) C3 into C4 (PEP carboxylase (PEPCL)), and iv) C4 into C3 (PEP carboxykinase (PEPCK), malic enzyme). Moreover, the $\mathrm{C} 3 / \mathrm{C} 4$ interconversions include either reversible reactions (2PG/PEP and $\mathrm{OAA} /$ malate inter-conversion) or irreversible but opposite reactions (PEP/pyruvate and PEP/OAA interconversions). The result is a dense sub-network of reactions that provides alternative pathways for the same conversion [28] and the occurrence of potential substrate cycles [29].

\section{Identification of the sub-network operating during methylotrophic growth}

The functional structure of the metabolic network operating during pure methylotrophic growth conditions of M. extorquens, i.e. growth on methanol as sole source of carbon and energy, was established by determining the sub-network of the GS metabolic model that includes all the reactions operating on methanol, thereafter referred to as the 'methylotrophic network' (Additional file 1). The identification of the methylotrophic and non-methylotrophic reactions was based on both theoretical and experimental considerations, including i) physiological parameters ii) genetic and biochemical data, iii) omics data (transcriptomic, proteomic, metabolomics), and extensive refinement. The reactions were confronted against all above criteria and were included or excluded a based on multi-criterion consideration and their overall score (Additional file 7). This reduction step was also a pre-requisite for in silico analysis of the methylotrophic network. The size of the GS model was too big to apply Elementary Flux Mode (EFM) analysis, a powerful tool to analyze the functional properties of metabolic networks from their topology [30], due to computational limitation. The Additional file 1 shows the diagram of the reduction process which was carried out in the following manner:

\section{i) Methylotrophic growth of M. extorquens}

The determination of the methylotrophic network was performed here to account for conditions where $M$. 


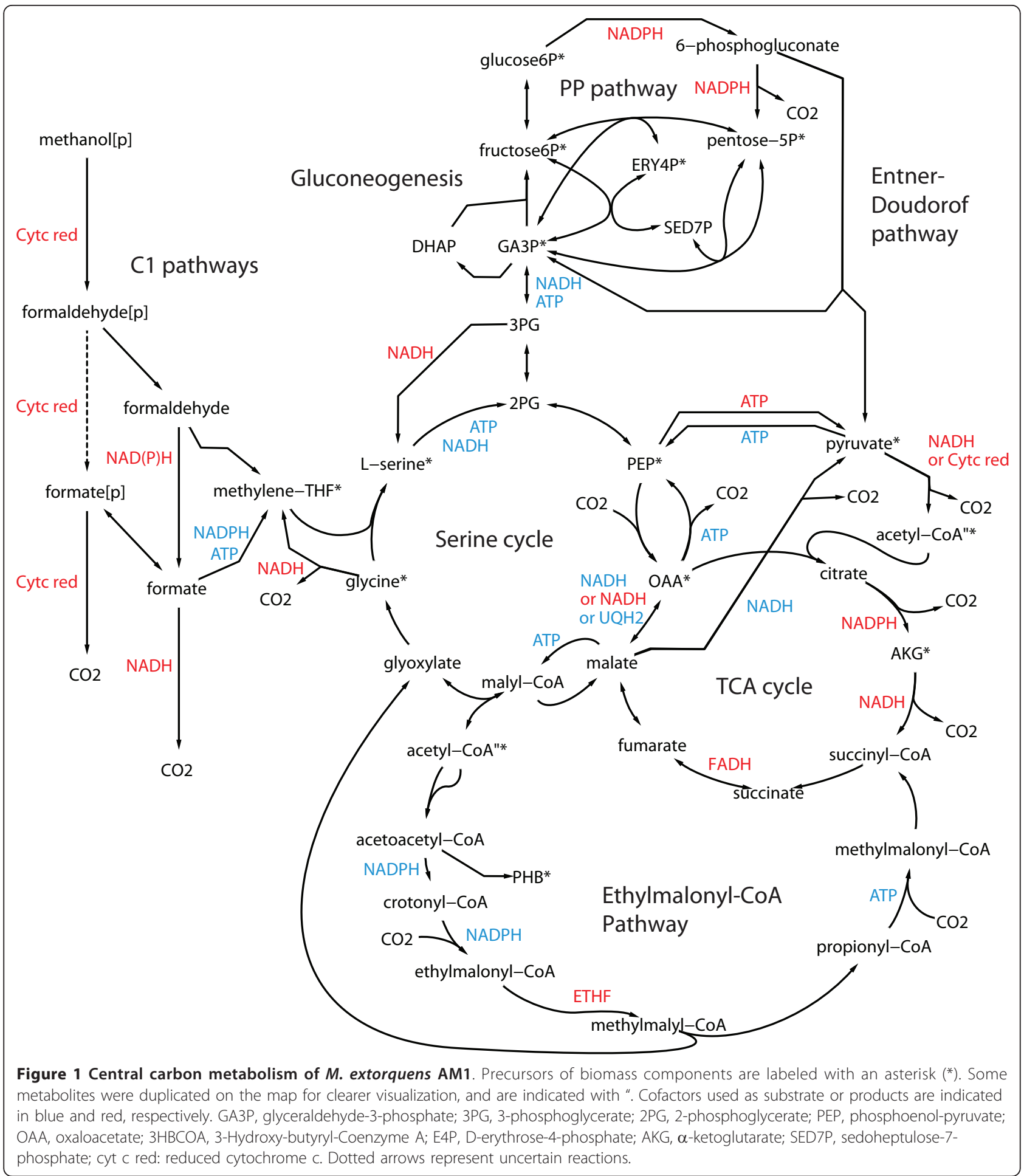

extorquens cells were grown exponentially in a minimal medium containing methanol as the sole added source of carbon and energy, $\mathrm{NH}_{4} \mathrm{Cl}$ as nitrogen source, and mineral salts, as described in material and methods. Such growth conditions allowed three levels of reduction of the GS metabolic model. First, the processes (transport and biochemical reactions) associated with the utilization of compounds (e.g. carbon sources, nitrogen sources, etc) included in the GS network but not occurring in the medium were removed. Similarly, the pathways and transport systems associated with metabolic end-products included in the GS network but not 
detected experimentally during methylotrophic growth were removed. Quantitative ${ }^{1} \mathrm{H}-\mathrm{NMR}$ analysis of culture supernatants collected after methylotrophic growth indicated that very few by-products accumulated in the medium, and only at negligible levels, allowing the removal of 187 reactions from the GS model. It was also assumed that in exponentially-growing cells no biomass degradation occurred, resulting in a further simplification of the network by removing the biochemical pathways specifically involved in the degradation or salvage of macromolecular components. This simplification resulted in the removal of 213 biochemical reactions. Some reactions associated with macromolecule degradation could be potentially involved in other metabolic processes, such as cofactor biosynthesis or recycling of anabolic by-products. Some of these reactions - a total of 13 - appeared to be relevant for growth on methanol and were kept in the methylotrophic network.

\section{ii) Genetic and biochemical data}

Literature data were used to further substantiate the methylotrophic network. More particularly, the phenotypes of gene deletion mutants were used to support the reduction process. From currently available literature, a total of 47 genes were shown to be essential during growth on methanol. Some of these genes encode multifunctional enzymes, so that a total of 51 biochemical reactions were associated with the 47 essential genes. All monofunctional enzymes (42 reactions) were kept in the methylotrophic model. For multifunctional enzymes, it could not be determined at this stage which reaction (s) was (were) responsible for essentiality, and other considerations were applied before making a decision as regard to their inclusion or exclusion of the methylotrophic network. In total, 2 reactions were excluded from genetic data analysis.

The enzyme assays available in the literature were considered to confirm the presence of biochemical reactions during methylotrophic growth, as well as their differential activities upon non-methylotrophic condition. Additional biochemical information from in vitro assay of particular reaction like spontaneous reaction or biochemical information in other microorganisms was used to validate reaction occurrence. In total, 13 reactions were excluded from biochemical data analysis.

\section{iii) Omics data}

The next reduction step was based on the comparison of omics data - including transtriptomics $[17,31]$, proteomics [31], and metabolomics data [17,32-35], collected for both methylotrophic-grown and nonmethylotrophic grown $M$. extorquens cells [17]. The molecular components corresponding to each type of omic data - e.g. protein for proteomics data - were kept in the methylotrophic network when they were identified to occur in methanol-grown cells, and/or their content was significantly higher - at least twice higher than in non-methanol-grown cells, i.e. succinate-grown cells. The score of components identified from transcriptomics (differential expression), proteomics (spectral counting, differential expression) and metabolomics (identification) were assigned to their corresponding biochemical reactions via the GPR association. Taken together, the omics data were involved in the confirmation of the occurrence of 175 reactions, and the exclusion of 296 reactions. The final methylotrophic network contained 717 reactions and 722 metabolites, associated with 706 genes (Table 1, Additional file 7), and included approximately two thirds of the components of the GS network. To validate the topology of the methylotrophic network, non methylotrophic reactions were constrained to zero in the stoichiometric model of $M$. extorquens metabolism, and the reduced model was used to simulate growth performance on methanol. FBA simulations showed that the network supports a theoretical maximal growth rate of $0.20 \mathrm{~h}^{-1}$, which is consistent with experimental values [36]. Moreover, this value was close to the maximal growth rate calculated with the GS network $\left(0.21 \mathrm{~h}^{-1}\right)$, indicating that no significant growth capacities were lost during the reduction of the GS network and furthermore suggesting that about one-third of the total metabolic potential of the bacterium is not required for growth on methanol.

\section{Dissimilation capabilities of the methylotrophic network}

The capability of methylotrophs to use methanol as sole energy and carbon source relies on the occurrence of both dissimilatory and assimilatory pathways, which fulfill all energetic and biosynthetic requirements, respectively. The reconstruction of the metabolic network of $M$. extorquens gave the opportunity to analyze the (system-level) organization of the two types of processes in this model methylotroph. Elementary flux mode (EFM) analysis, a powerful tool to analyze the functionality of metabolic networks from their topology [30], and FBA simulations, were carried out. Dissimilation and assimilation processes were first analyzed separately.

Dissimilation processes were defined here as processes resulting in the net conversion of methanol into $\mathrm{CO}_{2}$ and allowing energy conservation. The main dissimilation route is known to be the stepwise oxidation of methanol to $\mathrm{CO}_{2}$ using dedicated $\mathrm{C} 1$ pathways $[5,15]$. This process involves the periplasmic oxidation of methanol into formaldehyde, which is further oxidized to $\mathrm{CO}_{2}$ in the cytoplasm (see Figure 1). In this process one cytochrome $\mathrm{C}$ and two nicotinamide adenine dinucleotide (phosphate) $(\mathrm{NAD}(\mathrm{P}) \mathrm{H})$ are released, assuming the pyridine nucleotide dependent formate dehydrogenase (FDH) operates. This 'cytoplasmic' route can fulfill both adenosine triphosphate (ATP) and redox 
requirements at the same time. In case cytochrome $\mathrm{C}$ and NADH are reoxidized by the most effective oxidative phosphorylation mechanisms, a maximal yield of 5 ATP/methanol is predicted (Table 3). The additional potential routes for methanol dissimilation within the methylotrophic network could be detailed from the in silico investigations (Table 3). A periplasmic route of formaldehyde oxidation can be predicted in case methanol-dehydrogenase-like enzyme XoxF would act together with a periplasmic formate dehydrogenase [37]. Such a route would not generate $\mathrm{NAD}(\mathrm{P}) \mathrm{H}$ and hence could only fulfill ATP requirements, albeit with reduced maximal yield ( $3 \mathrm{ATP} /$ methanol). The network contains also other potential alternative mechanisms for the complete oxidation of methanol in which multi-carbon compounds are first generated and then completely oxidized to $\mathrm{CO}_{2}$. These processes would result from the combined action of $\mathrm{C} 1$ assimilation pathways (enabling the formation of multi-carbon compounds) and of catabolic pathways where multi-carbon compounds are fully oxidized to $\mathrm{CO}_{2}$ (via the TCA cycle or other decarboxylation reactions). The latter processes are the main energy conservation mechanisms upon utilization of $\mathrm{C} 2$ and other multi-carbon compounds, and are likely to be down-regulated during pure methylotrophic growth conditions. Nevertheless, the in silico analysis shows that the methylotrophic network contains the potential for these indirect dissimilation routes. They are however not efficient for energy conservation (Table 3 ) and are unlikely to operate upon methylotrophy from the energetic point of view.

\section{Assimilatory processes}

The consequences of the particular organization of primary $\mathrm{C} 1$ assimilation in $M$. extorquens AM1 were analyzed by examining the processes allowing the conversion of methanol into each of the 13 key carbon precursors, including $\mathrm{C} 1$ (Me-THF), C2 (acetyl-CoA, glycine), C3 (L-serine, pyruvate, PEP, glyceraldehyde-3phosphate), C4 (OAA, D-erythrose-4-phosphate (E4P), 3HBCOA), C5 ( $\alpha$-ketoglutarate, D-ribose-5-phosphate) and C6 (D-glucose-6-phosphate (G6P)). The number of EFMs ranged from 2018 to 6576 for the various carbon precursors (Table 4, Additional file 8). The serine cycle was involved in all assimilatory EFMs except for MeTHF, which can be also generated directly in the $\mathrm{C} 1$ pathways. This observation was consistent with the key role of the serine cycle pathway in methanol assimilation. The EMCP was involved in 93\%, 95\%, and 92\% of the EFMs generating Me-THF, acetyl-CoA, and (R)-3HBCOA, respectively. For all other carbon precursors, including the serine cycle intermediate glycine and Lserine, all assimilatory EFMs required the EMCP. These data emphasize the critical role of the EMCP (12 reactions), in addition to the $\mathrm{C} 1$ pathways (10 reactions) and the serine cycle (9 reactions), in methanol assimilation. Hence, the initial steps of $\mathrm{C} 1$ assimilation require the consecutive but obligatory operation of a high number of reactions. The minimal EFM length, representing the smallest number of reactions needed to convert methanol into each carbon precursor, was calculated for each of the 13 carbon precursors. The conversion of methanol into $\mathrm{C} 3$ compounds required at least 50 reactions, and the minimal number of reactions required to convert methanol into E4P was 63. Even for Me-THF and acetyl-CoA, the minimal EFM lengths were high (20 and 35 , respectively). These data indicated that the primary assimilation processes, ensuring the conversion of methanol into carbon precursors, is a particularly complex process in $M$. extorquens AM1. Despite the complexity of methanol assimilation, the carbon precursors are produced from methanol with carbon yields that are similar to that observed on glucose for species like $E$. coli and C. glutamicum.

\section{Interdependencies of dissimilatory and assimilatory processes}

The energetic efficiency of dissimilation processes determines the amount of energy available for assimilation and hence is a critical parameter of methylotrophic growth. The complete set of EFMs (152872) through the methylotrophic network was analyzed to investigate the relationships between dissimilation and assimilation processes. The EFMs were classified according to their biomass yields, and then to the various types of dissimilatory processes (Figure 2). In the EFM with the optimal

Table 3 Dissimilatory processes in the methylotrophic network.

\begin{tabular}{|c|c|c|c|}
\hline & $\max$ ATP & $\max \mathrm{NADH}$ & max NADPH \\
\hline Dissimilation processes & \multicolumn{3}{|c|}{ in mol.mol(methanol)-1 } \\
\hline $\mathrm{MeOH}->\mathrm{CO} 2$ (cytoplasmic FDH) & 5 & 2 & 2 \\
\hline $\mathrm{MeOH}->\mathrm{CO} 2$ (periplasmic FDH) & 3 & 0 & 0 \\
\hline $\mathrm{MeOH}+\mathrm{CO} 2$ (Ser cycle) - > Acetyl-CoA - > TCA cycle - > 2 CO2 & 1 & 0.5 & 0.5 \\
\hline $\mathrm{MeOH}+$ n CO2 (Ser cycle + EMCP) - > other C2s, C3s, etc - > central pathways - > n+1 CO2 & 1 & 0.5 & 0.5 \\
\hline
\end{tabular}

Types and number of dissimilatory EFMs detected in the methylotrophic network. For each type of dissimilation process, the theoretical maximum yields in ATP, $\mathrm{NADH}$ or NADPH are given. 
Table 4 Primary assimilation processes in the methylotrophic network.

\begin{tabular}{|c|c|c|c|c|c|c|}
\hline $\begin{array}{l}\text { compound/precursor } \\
\text { biosynthesis }\end{array}$ & $\begin{array}{l}\text { number of carbon in } \\
\text { precursor }\end{array}$ & $\begin{array}{l}\text { number of } \\
\text { EFMs }\end{array}$ & $\begin{array}{l}\text { Max. } \\
\text { molar-Yield }\end{array}$ & $\begin{array}{c}\text { Max. } \\
\text { carbon-Yield }\end{array}$ & $\begin{array}{l}\text { Minimal EFM } \\
\text { length }\end{array}$ & $\begin{array}{c}\text { EMCP } \\
\text { utilisation }\end{array}$ \\
\hline $\begin{array}{c}\text { 5, 10-methylenetetrahydrofolate } \\
\text { (Me-THF) }\end{array}$ & 1 & 2018 & 1.00 & 1.00 & 20 & $93 \%$ \\
\hline acetyl-CoA & 2 & 2440 & 0.45 & 0.91 & 35 & $95 \%$ \\
\hline glycine & 2 & 2054 & 0.42 & 0.84 & 62 & $100 \%$ \\
\hline L-serine & 3 & 2162 & 0.29 & 0.88 & 62 & $100 \%$ \\
\hline $\begin{array}{l}\text { D-glyceraldehyde-3-phosphate } \\
\text { (GA3P) }\end{array}$ & 3 & 2592 & 0.27 & 0.81 & 54 & $100 \%$ \\
\hline phosphateenolpyruvate (PEP) & 3 & 3390 & 0.32 & 0.97 & 53 & $100 \%$ \\
\hline pyruvate (PYR) & 3 & 3065 & 0.33 & 1.00 & 50 & $100 \%$ \\
\hline oxaloacetate (OAA) & 4 & 5366 & 0.32 & 1.29 & 51 & $100 \%$ \\
\hline $\begin{array}{l}\text { (R)-3-hydroxybutanoyl-CoA } \\
\text { (3HBCOA) }\end{array}$ & 4 & 2789 & 0.21 & 0.83 & 37 & $92 \%$ \\
\hline D-erythrose-4-phosphate (E4P) & 4 & 6576 & 0.20 & 0.81 & 63 & $100 \%$ \\
\hline$\alpha$-ketoglutarate & 5 & 3806 & 0.20 & 1.02 & 54 & $100 \%$ \\
\hline D-ribose-5-phosphate & 5 & 4663 & 0.16 & 0.81 & 61 & $100 \%$ \\
\hline D-glucose-6-phosphate & 6 & 2592 & 0.14 & 0.68 & 58 & $100 \%$ \\
\hline
\end{tabular}

Number and properties of primary assimilatory EFMs detected in the methylotrophic network. The EFMs correspond to the processes allowing the conversion of methanol into 13 key carbon precursors

biomass yield $\left(0.42 \mathrm{~g} \cdot \mathrm{g}^{-1}\right), 70 \%$ of methanol was directly oxidized via $\mathrm{C} 1$ pathways and the remaining was used for assimilation purposes. In a significant number of assimilatory EFMs, methanol was entirely oxidized to $\mathrm{CO}_{2}$ via the $\mathrm{C} 1$ pathways (Figure 2), meaning that no reduced carbon entered assimilatory pathways, and indicating that biomass could be fully generated from $\mathrm{CO}_{2}$. The highest biomass yield that could be obtained by such process was $0.283 \mathrm{~g} \cdot \mathrm{g}^{-1}$ (EFM number 122591). In this EFM, (Figure 3A), carbon fixation is achieved by a process involving both the EMPC and the serine cycle. The process starts in the EMCP where two glyoxylate molecules are generated from one acetyl-CoA and two $\mathrm{CO}_{2}$. The two glyoxylate

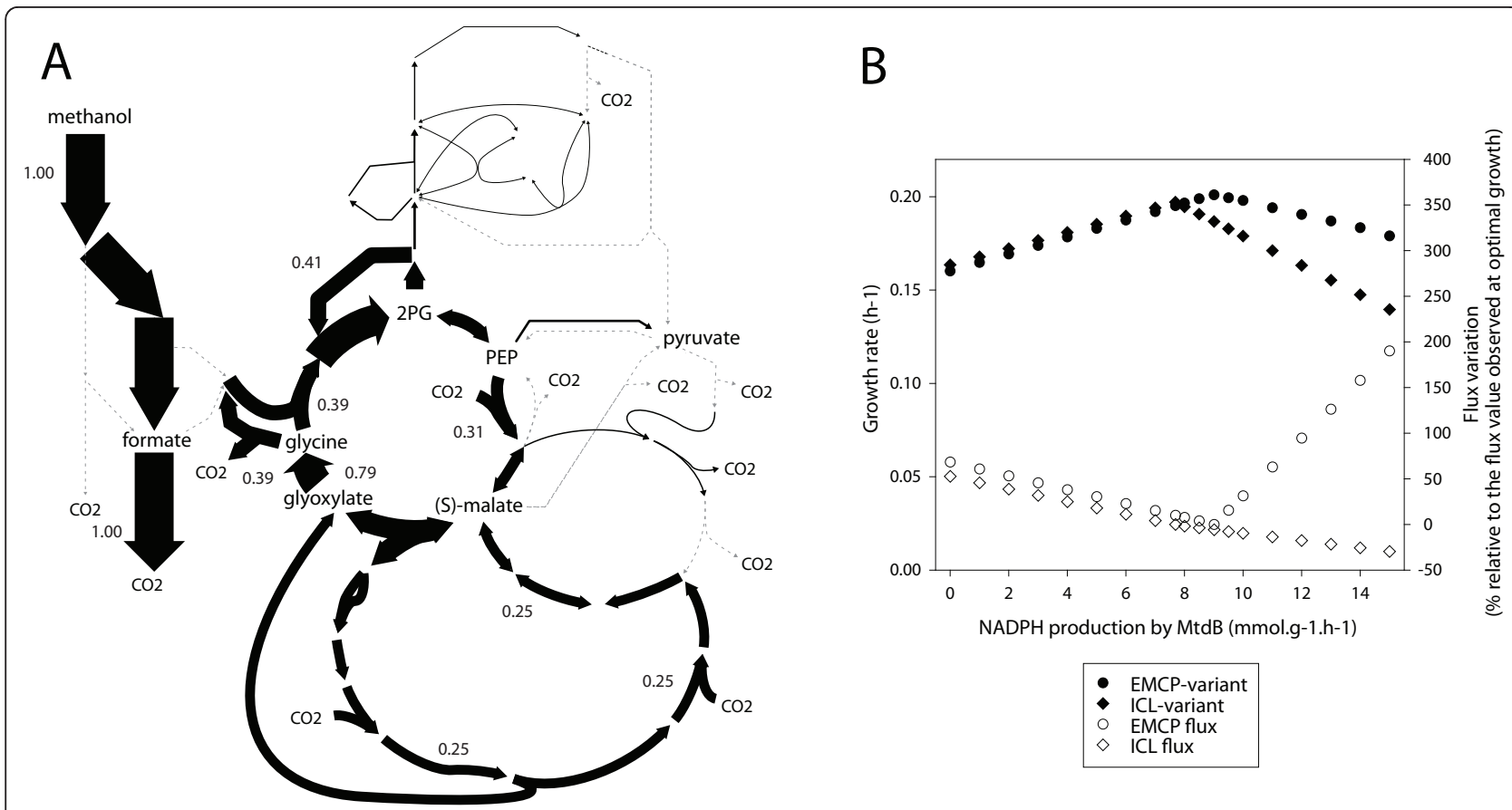

Figure 2 Structural EMCP properties compared to ICL variant. A. EFM (\#122591) with the optimal biomass yield among the EFMs where biomass carbon is derived exclusively from $\mathrm{CO}_{2}$. B. FBA simulation of optimal growth rate depending upon a fixed proportion of NADPH/NADH produced by methylene- $\mathrm{H}_{4}$ MPT dehydrogenases MtdA and MtdB in EMCP and ICL-variant. 


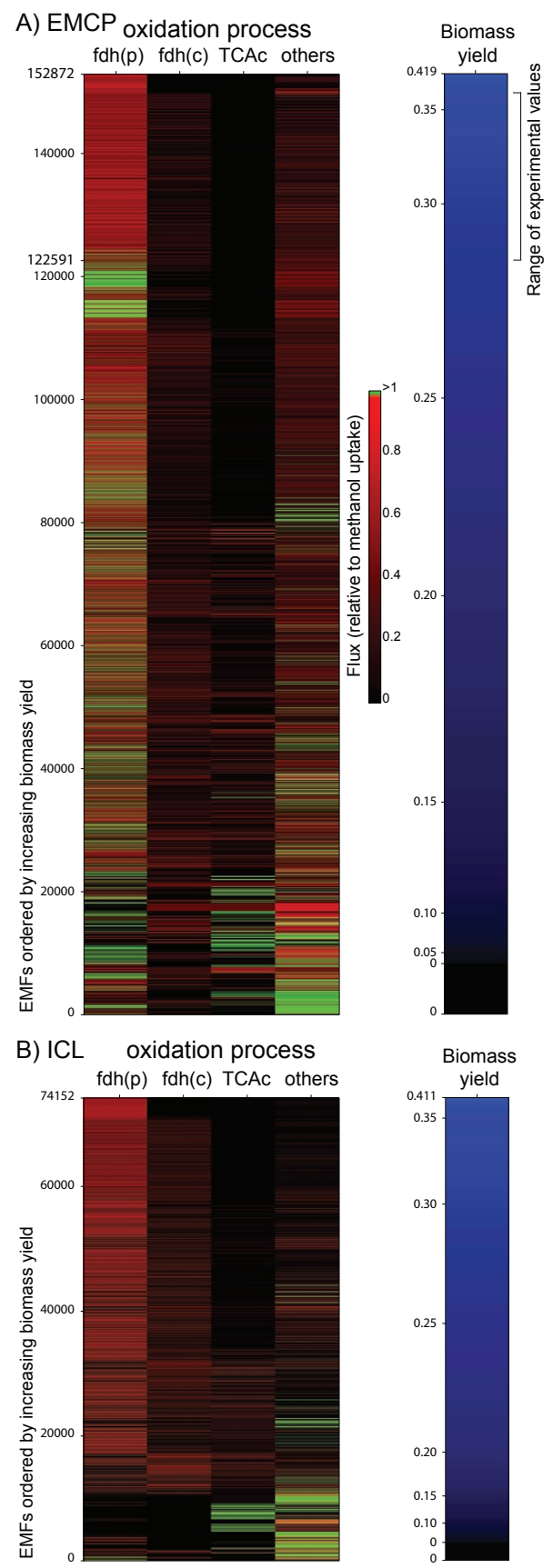

Figure $3 \mathrm{EFM}$ analysis of the balance between dissimilation and assimilation in EMCP and ICL variants. The biomass-forming EFMs were calculated and sorted according to the biomass yield (from bottom to top, blue color scale). For each EFM, the flux through the main dissimilatory processes (see text for details) were extracted and plotted separately. Fluxes were expressed relative to the rate of methanol uptake, and were plotted using a colour-scale (black to green). First lane: cytoplasmic FDH, fdh(c); second lane: periplasmic FDH, fdh(p); third lane: TCA oxidation of acetyl-CoA, TCAC; fourth lane: other dissimilation process. A: EFMs calculated with the methylotrophic network, which contains the ethylmalonylCoA pathway (EMCP). B: EFMs calculated with the network variant where EMCP was replaced by the glyoxylate cycle (ICL variant). molecules enter the serine cycle to produce two glycine molecules. One glycine is converted by the glycine cleavage complex into one $\mathrm{CO}_{2}$ and one Me-THF. The latter compound allows the conversion of the second glycine molecule into serine, which is used in subsequent steps of the serine cycle, allowing both the regeneration of the initial acetyl-CoA molecule and enabling through the operation of the entire mechanism - the formation of all carbon precursors needed for biosynthetic purposes. The overall carbon balance is $2 \mathrm{CO}_{2} \rightarrow$ 1 glyoxylate. As this process requires the release of $\mathrm{Me}-$ THF via the glycine decarboxylase complex, it represents a distinct feature compared to the classical operation of the serine cycle. The carbon yield of the $\mathrm{CO}_{2}-$ assimilation process is significantly lower than observed for methanol assimilation (40\% vs $62 \%)$. The ATP needs are twice higher $(7.2 \mathrm{vs} 3.8 \mathrm{~mol} \cdot \mathrm{mol}$ (carbon assimilated) $)^{-1}$ ), and the redox needs are two to three times more elevated. Such high energetic costs can be covered by methanol oxidation, but the overall $\mathrm{CO}_{2}$ assimilation process is much less favorable than methanol assimilation. This $\mathrm{CO}_{2}$ assimilation process was not reported so far and is a direct consequence of the capability of the EMCP to ensure $\mathrm{CO}_{2}$ fixation.

\section{Substitution of the ethylmalonyl-CoA pathway by the glyoxylate cycle}

The recently discovered EMCP is an alternative to the classical glyoxylate cycle for the biosynthesis of glyoxylate from acetyl-CoA in organisms lacking isocitrate lyase (ICL) $[18,19,38]$. To compare the metabolic properties conferred by the EMCP with that of the glyoxylate cycle, we generated a variant of the methylotrophic network lacking the EMCP but possessing the glyoxylate cycle. This was done by setting to zero the flux from crotonyl-CoA to propionyl-CoA and by adding the ICL reaction. Malate synthase, the enzyme of the glyoxylate cycle that catalyzes the condensation of acetyl-CoA and glyoxylate into malate, was not added since $M$. extorquens can use a combination of two enzymes to achieve the same reaction [39], as described also in R. sphaeroids [40]. As expected, the glyoxylate cycle was essential for methanol growth and was found in all assimilatory EFMs. The maximal biomass yield predicted for the ICL variant $\left(0.41 \mathrm{~g} \cdot \mathrm{g}^{-1}\right)$ was similar to that observed for the EMCP variant yield $\left(0.42 \mathrm{~g} \cdot \mathrm{g}^{-1}\right)$. To obtain such maximal growth, the rate of methanol oxidation in the ICL variant was smaller (61\% vs $70 \%)$ and the NADPH requirements lower than in the EMCP variant, showing a higher energetic efficiency of the ICL variant. In contrast to the EMCP, the ICL variant was not found to allow entire biomass formation from $\mathrm{CO}_{2}$.

To investigate the potential role of the EMCP in redox balancing, we compared the capability of the two 
metabolic network variants to respond to varying NADPH production levels. FBA simulations were performed where the amount of NADPH produced by the Me- $\mathrm{H}_{4} \mathrm{MPT}$ dehydrogenase MtdB [41], which can use either NADH or NADPH, was varied from 0 to 15.0 $\mathrm{mmol} \cdot \mathrm{g}^{-1} \cdot \mathrm{h}^{-1}$ (0 to $100 \%$ of MtdB flux). For both the EMCP and ICL variants, the absence of NADPH production in the $\mathrm{C} 1$ pathways can be compensated by other NADPH-production systems, the PPP or malic enzyme. The theoretical maximal growth rate increased with NADPH production until a maximum is reached, which represents the optimal balance between NADPH production and growth. Maximal growth for the EMCP variant was obtained at higher NADPH production level than the ICL variant $\left(9.0 \mathrm{vs} 7.7 \mathrm{mmol} \cdot \mathrm{g}^{-1} \cdot \mathrm{h}^{-1}\right)$, in agreement with the higher redox demand identified previously. The EMCP variant was able to maintain higher growth rates when the NADPH production was further increased. This capability was correlated with a significant increase of the EMCP flux. The increase in the EMCP flux was accompanied by the truncation of the serine cycle. Rather than being converted to OAA, PEP is converted into pyruvate via pyruvate kinase, which is further converted by pyruvate dehydrogenase into acetyl-CoA, which enters the EMCP. This pathway generates ATP (via pyruvate kinase) and releases NADH (via pyruvate dehydrogenase), resulting in a transhydrogenase-like mechanism where the redox equivalents are transferred from NADPH to $\mathrm{NAD}^{+}$in addition to the transfer to $\mathrm{CO}_{2}$.

\section{Fragility of the methylotrophic network}

Robustness is an inherent property of a metabolic network and is defined as the capability of this network to operate despite one - or more - reactions are removed. The robustness of the methylotrophic network was analysed using minimal cut sets (MCSs), which correspond to minimal combinations - singlets, pairs, triplets, etc of reactions whose removal blocks the operation of a target metabolic function [42]. The identification of all MCSs in a metabolic network allows the calculations of the fragility coefficient (FC) of each reaction. The FC of a reaction represents the probability that the metabolic system fails to achieve the target function when the reaction is removed. This approach was applied to analyze the robustness of the methylotrophic network using growth as the target metabolic function. A significant number of reactions (391) were found to have a FC of 1 and are therefore predicted to be essential (Additional file 9). Among these 391 reactions, 30\% are catalyzed by multiple enzymes, indicating that enzyme redundancy is significantly used to avoid metabolic resilience in $M$. extorquens AM1. Most of the essential reactions (279) were found in biosynthetic pathways, which is consistent with studies performed with other organisms. The FCs of reactions found in central metabolism spanned over a wide range of values but distributed heterogeneously among metabolic pathways (Figure 4). Some processes, such as the C1- and carbohydrate pathways, and C3/C4 interconversion reactions, had low FCs and hence were predicted to be robust parts of the metabolism. Most other parts of the central metabolism had high FCs and hence were predicted to be fragile. Of the 84 reactions of the central carbon metabolism, 40 were found to be essential. The essential reactions concentrated in the primary assimilation processes (Figure 4). Most reactions of the serine cycle (67\%) and of the EMCP (100\%) were predicted to be essential for methylotrophic growth, indicating the assimilation processes to be highly fragile. The same observation holds true for gluconeogenesis. Because a particular reaction can be catalyzed by one or several enzymes, the essentiality of a reaction does not necessarily mean that the removal by gene deletion - of one particular enzyme will be lethal. Among the 40 essential reactions found in central pathways, 29 were catalyzed by a single enzyme. Accordingly, 19 of these genes have been studied experimentally, and $95 \%$ of them were shown to be lethal for methylotrophic growth [43] (Additional file 10). For an essential reaction with multiple enzymes, one isoenzyme can compensate the lack of the other one(s). Genes encoding isoenzymes are therefore predicted to be not essential. However, mutant analysis showed that 4 out of 11 essential reactions with multiple enzymes found in primary assimilation processes, were encoded by gene where deletion were lethal for growth on methanol. This observation suggests that the products of these genes play an essential role during growth on methanol that cannot be compensated by the other potential enzymes catalysing the same reactions, or that they have different regulations, or both. Taken together, these data showed that the main processes of methanol assimilation are highly fragile in $M$. extorquens AM1. The robustness of the metabolism of $M$. extorquens AM1 was also calculated for a $\mathrm{C} 2$ compound (acetate), and for a C4 compound (succinate). The data were compared to that calculated for $E$. coli using the model of Klamt et al. [42,44]. The utilization of $\mathrm{C} 2$ compounds like acetate relies on the EMC pathway in M. extorquens and on the glyoxylate cycle in E. coli. Interestingly, the metabolic robustness of the central metabolism upon acetate growth was calculated to be lower in the former organism compared to the latter. Upon growth on succinate, which does not rely on $\mathrm{C} 1$-associated pathways but on common pathways (e.g. TCA cycle) that are similar in the two organisms, metabolic robustness was calculated to be similar between the two organisms. These observations suggest that metabolic fragility in $M$. 


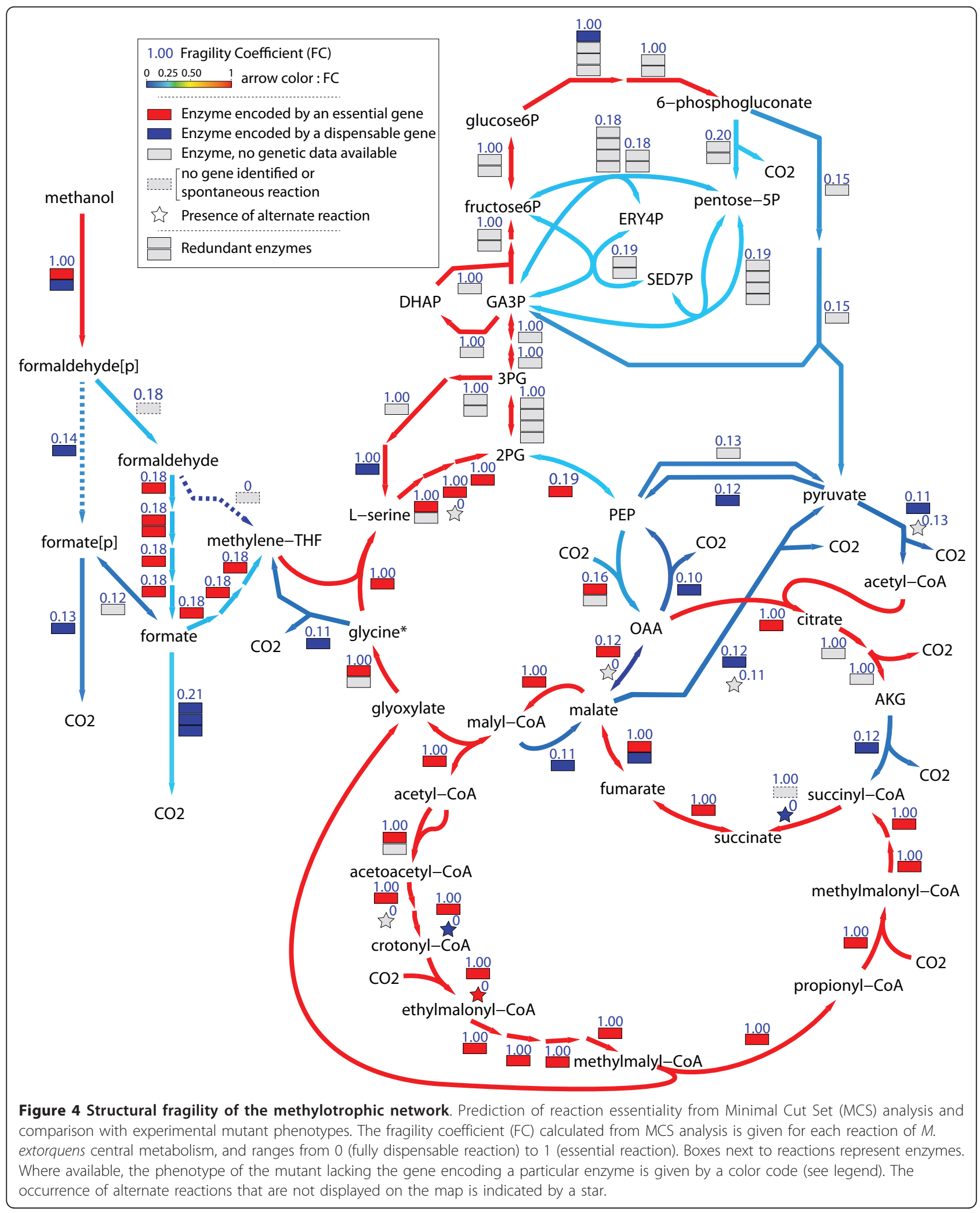


extorquens is mainly related to the nature and organization of $\mathrm{C} 1$ (\& $\mathrm{C} 2)$ pathways in this organism.

\section{Distribution of metabolic fluxes during methylotrophic growth}

The distribution of metabolic fluxes during methylotrophic growth was determined using ${ }^{13} \mathrm{C}$-metabolic flux analysis $\left({ }^{13} \mathrm{C}\right.$-MFA). ${ }^{13} \mathrm{C}$-MFA was already successfully applied to $M$. extorquens AM1, which provided valuable insights into the operation of central pathways [8]. The novel insights such as the operation of the EMCP [19], the organization of central assimilatory pathways [17], and the biomass composition (this study) necessitates the reinvestigation of metabolic flux analysis in $M$. extorquens AM1 during growth on methanol. To this end, a series of ${ }^{13} \mathrm{C}$-methanol labeling experiments was performed, and the isotopic information was monitored by both mass spectrometry and two dimensional nuclear magnetic resonance (2D-NMR) [45] (Additional file 11, 12). Such analytical combination provides critical information for the resolution of central pathways [19]. The flux distribution obtained from these investigations is displayed in Figure 5 and listed in Additional file 13, and the fitting accuracy and sensitivity analysis are listed in Additional files 12, 13, 14 and 15. The flux data obtained in the present were significantly different from that previously published [8]. This is mainly due to the major advances made in the meantime in the description and understanding of $M$. extorquens metabolism, and it is now known that the size and topology - and hence the possible carbon flows - of central carbon metabolism are more complete and much different than previously assumed. For this reason they can be hardly compared.

The flux data of Figure 5 indicated that $12.7 \pm 0.6 \mathrm{mmol}$ - $\mathrm{g}^{-1} \cdot \mathrm{h}^{-1}$ of methanol, $84 \%$ of methanol consumed $\left(15.10 \pm 0.60 \mathrm{mmol} \cdot \mathrm{g}^{-1} \cdot \mathrm{h}^{-1}\right)$, was directly oxidized to $\mathrm{CO}_{2}$ within the $\mathrm{C} 1$ pathways. A release of $\mathrm{CO}_{2}$ was also observed within biosynthetic pathways $(2.6 \%$ of consumed methanol) and central metabolism (5.4\%). The release of $\mathrm{CO}_{2}$ in central metabolism was due to substantial fluxes through malic enzyme $\left(0.36 \mathrm{mmol} \cdot \mathrm{g}^{-1}\right.$. $\left.\mathrm{h}^{-1}\right)$ and PEPCK $\left(0.26 \mathrm{mmol} \cdot \mathrm{g}^{-1} \cdot \mathrm{h}^{-1}\right)$, but was not associated with dissimilation processes. Indeed, the TCA contributed to only $1.1 \%$ of total $\mathrm{CO}_{2}$ release and operated in an incomplete and anabolic mode. The flux of $\mathrm{C} 1$ assimilation via Me-THF was $2.4 \pm 0.02 \mathrm{mmol} \cdot \mathrm{g}^{-1}$. $\mathrm{h}^{-1}$, which represents $16 \%$ of methanol uptake. The flux data clearly showed the central role of the serine cycle in distributing the carbon flow throughout the entire metabolic network to fulfill the requirements in carbon precursors. About $20 \%\left(0.5 \mathrm{mmol} \cdot \mathrm{g}^{-1} \cdot \mathrm{h}^{-1}\right)$ was directed towards gluconeogenesis and carbohydrate pathways, and $30 \%$ was routed to the formation of pyruvate and
TCA cycle intermediates. The release of acetyl-CoA by the serine cycle was significant $\left(1.80 \mathrm{mmol} \cdot \mathrm{g}^{-1} \cdot \mathrm{h}^{-1}\right)$. The major part $\left(1.4 \mathrm{mmol} \cdot \mathrm{g}^{-1} \cdot \mathrm{h}^{-1}\right)$ was recycled back to the serine cycle by the EMCP, and the remaining was used for anabolic purposes. The fixation of $\mathrm{CO}_{2}$ occurring within central pathways was calculated from the difference between $\mathrm{CO}_{2}$-utilizing and $\mathrm{CO}_{2}$-releasing fluxes, and was $2.4 \mathrm{mmol} \cdot \mathrm{g}^{-1} \cdot \mathrm{h}^{-1}$. This value was similar to the rate of methanol assimilation via Me-THF (2.4 $\left.\mathrm{mmol} \cdot \mathrm{g}^{-1} \cdot \mathrm{h}^{-1}\right)$. These data were consistent with the observation that $50 \%$ of the biomass carbon derived from $\mathrm{CO}_{2}[7,46]$. Such carbon balance can be obtained in case two molecules of glyoxylate are generated per turn of the EMCP [19], which requires that the propionyl-CoA produced in this pathway is not directly used for anabolic purposes but converted into glyoxylate. Accordingly, the replenishment of the glyoxylate pool from propionyl-CoA was almost identical to the direct release of glyoxylate within the EMCP $(0.70 \pm 0.02$ $\left.\mathrm{mmol} \cdot \mathrm{g}^{-1} \cdot \mathrm{h}^{-1}\right)$. No flux was found through the glycine cleavage complex, indicating that the $\mathrm{CO}_{2}$ assimilation mechanism identified from EFM analysis was not operating during pure methylotrophy, for chosen cultivation conditions. Surprisingly, a glycolytic flux through the Entner-Doudoroff pathway was observed. This flux (0.08 $\pm 0.02 \mathrm{mmol} \cdot \mathrm{g}^{-1} \cdot \mathrm{h}^{-1}$ ) was low compared to the rate of formate assimilation but was significant to fit the labeling data and represented about $14 \%$ of total pyruvate synthesis. This observation indicated that some carbon atoms were recycled through gluconeogenesis and glycolysis during methylotrophic growth.

The flux data provided valuable information regarding the $\mathrm{C} 3 / \mathrm{C} 4$ pathways. The synthesis of pyruvate, which is required for various anabolic purposes, was proposed earlier to proceed via the conversion of PEP into pyruvate, via pyruvate kinase [6]. The flux data showed that pyruvate was synthesized by three different routes, including pyruvate kinase, the ED pathway and malic enzyme. The main flux was carried out by malic enzyme $\left(0.36 \mathrm{vs} 0.13 \mathrm{mmol} \cdot \mathrm{g}^{-1} \cdot \mathrm{h}^{-1}\right)$. Moreover PEP synthase, which catalyses the reaction opposite to pyruvate kinase, is active and its flux $\left(0.13 \mathrm{mmol} \cdot \mathrm{g}^{-1} \cdot \mathrm{h}^{-1}\right)$ is higher than that of the latter enzyme. This observation indicated the occurrence of a substrate cycle between PEP and pyruvate due to the parallel activity of pyruvate kinase and PEP synthase, and in which $68 \%$ of pyruvate is recycled. Three additional substrate cycles were observed in this part of the metabolism. Two of them were related to $\mathrm{C} 3 / \mathrm{C} 4$ inter-conversions: i) PEP/OAA cycling via PEPCL and PEPCK (13\% of PEP recycled), and ii) $\mathrm{PEP} /$ malate/pyruvate cycling via PEPCL, malate dehydrogenase, malic enzyme and PEP synthase (4\% of PEP recycled via malate and pyruvate). The fourth substrate cycle was observed between malate and (acetyl- 


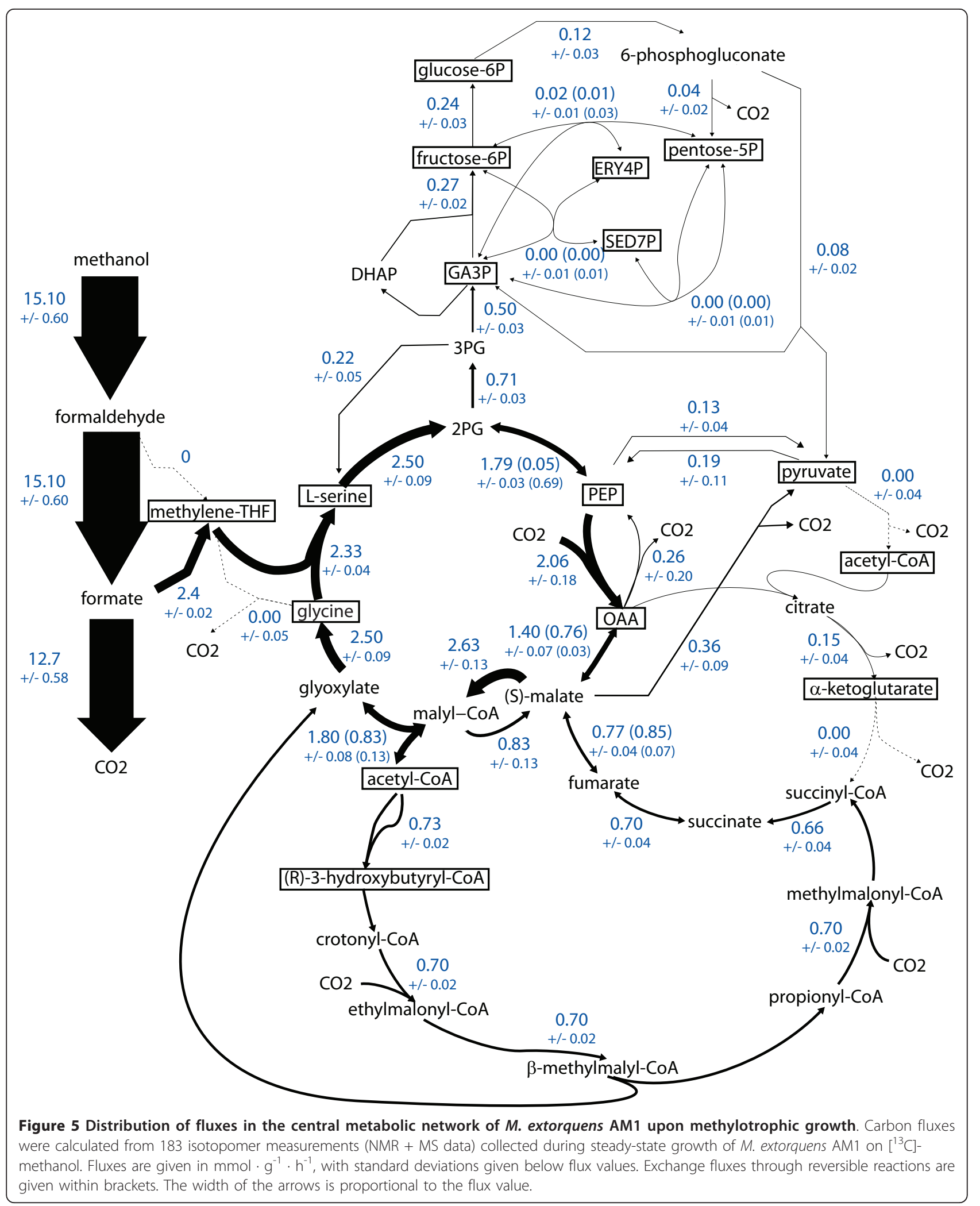


$\mathrm{CoA}+$ glyoxylate). It relies on the reversible activity of the malyl-CoA lyase [47] and on the parallel operation of malate-CoA ligase and malyl-CoA thioesterase. Malate-CoA ligase is responsible for the release of glyoxylate and acetyl-CoA from malyl-CoA in the serine cycle. Malyl-CoA thioesterase catalyzes the opposite reaction. This enzyme is supposed to play a key role during growth on multicarbon compounds, but its activity is not expected during methylotrophic growth. Indeed, the enzyme activity is down regulated during growth in the presence of methanol [48]. However, a significant activity of this enzyme is still detected upon methanol growth [48], and represents $22 \%$ of the activity found on acetate. Such level of activity is likely to be sufficient to maintain a flux in the reaction, thereby resulting in substrate cycling. The flux data show not only that the latter cycle operates in $M$. extorquens AM1 during growth on methanol, but also that the extent of recycling is significant $(32 \%)$. The energetic cost of metabolite recycling within the four above-mentioned processes was calculated from the flux data to be 1.3 mmol ATP $\cdot \mathrm{g}^{-1} \cdot \mathrm{h}^{-1}$, with the most expensive one $(0.8$ mmol ATP $\cdot \mathrm{g}^{-1} \cdot \mathrm{h}^{-1}$ ) being the malate/(acetyl-CoA +glyoxylate) cycle. The energetic cost was calculated to represent about $4 \%$ of the total production of ATP (29.3 ATP $\left.\mathrm{mmol} \cdot \mathrm{g}^{-1} \cdot \mathrm{h}^{-1}\right)$. This energetic expense might represent a tradeoff between optimal metabolic efficiency and the ability to switch metabolic modes.

The total demand in NADPH during methylotrophic growth could be calculated from the fluxes in NADPHutilizing reactions and biomass requirements, and was $5.6 \mathrm{mmol} \cdot \mathrm{g}^{-1} \cdot \mathrm{h}^{-1}$. The demand was mainly due for formate assimilation $\left(2.3 \mathrm{mmol} \cdot \mathrm{g}^{-1} \cdot \mathrm{h}^{-1}\right)$, biosynthetic requirements $\left(1.8 \mathrm{mmol} \cdot \mathrm{g}^{-1} \cdot \mathrm{h}^{-1}\right)$, and operation of the EMCP $\left(1.4 \mathrm{mmol} \cdot \mathrm{g}^{-1} \cdot \mathrm{h}^{-1}\right)$. The ${ }^{13} \mathrm{C}$-flux data showed also that the two NADPH-forming reactions found within central carbon pathways, i.e. isocitrate dehydrogenase and G6P dehydrogenase, contributed only negligibly (below 5\%) to the total NADPH production. Hence, most of NADPH is generated alongside formaldehyde oxidation. From these data it can be calculated that the production of NADPH in the $\mathrm{C} 1$ pathway should be $5.3 \mathrm{mmol} \cdot \mathrm{g}^{-1} \cdot \mathrm{h}^{-1}$ to close the NADP balance. To evaluate the ATP balance, flux variability analysis and FBA simulations were performed in which the methylotrophic network was constrained with the ${ }^{13} \mathrm{C}$ flux data, biomass requirements, experimental rates of growth and methanol uptake, and maintenance energy. Because the respiratory mechanisms by which the reduced cofactors (NADH, cytochrome $\mathrm{C}$ ) generated in the $\mathrm{C} 1$ pathways are not clearly established in $M$. extorquens the ATP cannot be firmly established from the flux data. Nevertheless, the simulations showed that, if dissimilation proceeds via the cytoplasmic, NADH- dependent route at maximal ATP efficiency (Table 3), then the total production of ATP $\left(44 \mathrm{mmol} \cdot \mathrm{g}^{-1} \cdot \mathrm{h}^{-1}\right)$ would be in large excess compared to the requirements $\left(29.3 \mathrm{mmol} \cdot \mathrm{g}^{-1} \cdot \mathrm{h}^{-1}\right)$.

\section{Discussion}

The genome scale metabolic network reconstructed in this work offers an integrated view of the current metabolic knowledge of the methylotrophic bacterium $M$. extorquens AM1. It provides new insights into the biochemistry of this organism and reveals the networkscale organization of metabolic processes as well as a first evaluation of the complete metabolic potential of this bacterium. The metabolic reconstruction allowed a detailed picture of the central carbon metabolism of the bacterium, which appears as a mosaic of common (TCA cycle, anaplerotic processes, gluconeogenesis, PPP, ED pathway) and specific (C1 pathways, serine cycle, $\mathrm{EMCP}$ ) pathways, enabling growth on $\mathrm{C} 1$ and multicarbon ( $\mathrm{C} 2$ to $\mathrm{C} 4)$ compounds. The core of the central metabolism is organized as a highly unusual series of tightly embedded metabolic cycles that operate as an entity to achieve $\mathrm{C} 1$ assimilation during methylotrophic growth. The ability to assimilate $\mathrm{C} 1$ compounds relies on a complex metabolic machinery, in which the initial steps - from methanol to biomass precursors - require a particularly high number of reactions (e.g. at least 36 reactions to obtain acetyl-CoA). The entire process is strongly reductive and energy-consuming. Most of these reactions require enzymes and cofactors that are specific to $\mathrm{C} 1$ - or $\mathrm{C} 2$ - growth, and must be biosynthesized for the purpose of $\mathrm{C} 1$ or $\mathrm{C} 2$ utilization. Hence, the energetic costs for the biosynthesis and maintenance of this machinery are likely to be substantial for the bacteria. In addition, the network-scale analysis reveals that $\mathrm{C} 1$ assimilation is structurally fragile. Similarly to other metabolically specialized microorganisms [49], the core metabolism of $M$. extorquens is characterized by a high fraction of reactions that are essential for methylotrophic growth (almost 50\%). In such networks robustness arise usually from enzyme (and genetic) redundancy, where multiple isoenzymes can catalyze essential reactions $[49,50]$. The genetic or biochemical deficiency in one isoenzyme can be compensated by another one. Accordingly, the percentage of multiple genes encoding essential reactions in specialized microorganisms is much higher than in generalist - or flexible - organisms such as E. coli, B. subtilis or S. cerevisiae (> $30 \%$ vs $10 \%$ redundancy, respectively) [50]. The high degree of redundancy (28\%) observed in $M$. extorquens confirms the specialized metabolism of this methylotroph. Furthermore, among the essential reactions with multiple genes, a significant number of particular genes were shown experimentally to be essential for growth 
on methanol, suggesting that the alternative gene(s) have different functions or regulations, hence are not functionally redundant. Therefore, the number of genes essential for methylotrophy is high, and the risk that a gene mutation results in loss of methylotrophic capacity is elevated. This could explain, in part, the successes in the identification of such genes in the last 2 decades [43]. The observations emerging from metabolic network analysis are highly consistent with experimental evolution experiments in which a significant number of clones collected after prolonged cultivation (1500 generations) on succinate lost their methylotrophic capacity [36]. Taken together, available data suggest that a selection pressure is required to maintain methylotrophy in $M$. extorquens, indicating that the bacterium encounters frequently methanol in its natural environment and that its usage provides critical advantage in terms of ecological competitiveness.

The metabolic reconstruction data indicate also that a dense network of $\mathrm{C} 3 / \mathrm{C} 4$ inter-conversions plays a critical role as a branch-point connecting specific and common pathways. Indeed, seven reactions interconnect three C3 (2PG, PEP, pyruvate) and two C4 (malate, OAA) intermediates, thereby strongly embedding the serine cycle, the TCA cycle, anaplerotic processes, and gluconeogenesis (via 2PG). Such topology allows a wide range of alternative metabolic routes and provides metabolic flexibility. Van Dien et al. $[6,28]$ have emphasized the role of these processes in the metabolism of multicarbon compounds. During growth on $\mathrm{C} 4$ compounds such as succinate, a functional TCA cycle is required and the generation of acetyl-CoA is ensured by pyruvate dehydrogenase. Hence, the conversion of $\mathrm{C} 4$ compounds into pyruvate is critical and can be achieved by redundant routes. During methylotrophic growth - in which a complete TCA does not operate -, the $\mathrm{C} 3 / \mathrm{C} 4$ inter-conversions primarily ensure the opposite conversion of $\mathrm{C} 3$ intermediates into $\mathrm{C} 4$ intermediates in the serine cycle. They provide also alternative metabolic routes for such conversions, as observed with the significant conversion of PEP into pyruvate via PEPCL, malate dehydrogenase, and malic enzyme, though the role of this pathway is still unclear. The most striking feature is, however, the occurrence of substantial substrate cycling within the C3/C4 inter-conversions upon growth on methanol. In addition, substrate cycling was also observed between C4 (malate) and C2 (glyoxylate + acetyl-CoA), at the branch-point between the serine cycle, the TCA cycle, the EMCP and anaplerosis. Substrate cycles are resulting from the simultaneous operation of - non reversible opposite reactions or processes, at the expense of energy. They can represent adaptation mechanisms allowing fast switching of metabolic processes $[17,29]$. The nature of the substrates cycles observed predicted during methylotrophic growth indicated that the entire set of reactions starting from PEP or pyruvate to acetyl$\mathrm{CoA}$ and glyoxylate are operating as a fully reversible process. As mentioned above, these processes are the branching-point of the specific - i.e. serine cycle, EMCP - and common - i.e. TCA cycle, anaplerotic processes, and gluconeogenesis - pathways. They are also the starting point of a large number of biosynthetic routes, and the entry point of the utilization pathways of all $\mathrm{C} 1, \mathrm{C} 2$ and $\mathrm{C} 3 / \mathrm{C} 4$ carbon sources used by the bacterium. Taken together, these data suggest that upon pure methylotrophic growth the occurrence of substrate cycling provides flexibility between specific and common pathways, thereby allowing fast switching of the metabolism from methanol to alternative carbon substrates.

Though $M$. extorquens AM1 is considered to be a methylotroph but not an autotroph, the in silico investigations revealed a potential of autotrophy in this bacterium, which relies on the unique property of the EMCP to enable $\mathrm{CO}_{2}$ fixation. The $\mathrm{CO}_{2}$-fixation mode involves a cyclic operation of both the EMCP and serine cycle to generate one glyoxylate from two $\mathrm{CO}_{2}$. It can potentially operate independently of methanol assimilation in $M$. extorquens, but was not observed during methylotrophic growth in our investigations. Because the genome of $M$. extorquens contains the complete information for a photosynthetic machinery, it is tempting to speculate that this bacterium may operate in a photoautotrophic mode. However, there is currently no experimental evidence of such behavior in $M$. extorquens. The question of the role, benefit and conservation of this pathway in $M$. extorquens and other organisms is still unclear. The EMCP is more complex and energy-demanding than the glyoxylate cycle though it provides a higher carbon balance for assimilation. In photosynthetic methanol utilizers, carbon dioxide functions as an electron sink for the excess electrons in methanol [51]. It was recently proposed that $\mathrm{CO}_{2}$ fixation could represent an alternative mechanism of cofactor recycling in bacteria [52]. The potential role of the EMCP in such mechanism was recently shown from investigations of $\mathrm{CBB}$ mutants defective for the reductive PPP pathway in acetategrown $R$. sphaeroides [53]. Our investigations show that the EMCP can potentially play such a role in $M$. extorquens upon methylotrophic growth. If further investigations are required to determine the actual physiological benefits of the EMCP in serine cycle methylotrophs, our investigations show that this pathway can potentially play the role of a redox-balancing mechanism or of an autotrophic pathway.

\section{Conclusions}

The unusual organization of the central carbon metabolism of M. extorquens AM1 allows efficient utilization of 
C1 compounds via highly specific - and fragile - pathways but is versatile enough around a flexible backbone of $\mathrm{C} 2 / \mathrm{C} 3 / \mathrm{C} 4$ inter-conversions to allow switching to other carbon sources. These observations showed that the bacterium maintains active metabolic processes that are not needed for methanol utilization but allow adaptation to other carbon sources. This hypothesis is consistent with the observation that methanol is produced by plant with methanol release in the morning $[54,55]$. This work emphasizes that the metabolism of the bacterium is adapted to its lifestyle not only in terms of enzymatic equipment, but also in terms of network-level structure and regulation. It suggests that the metabolism of the bacterium is adapted both structurally and functionally to an efficient but transitory utilization of methanol. This work illustrates that the combination of GS network modeling and experimental approaches provides novel insights into the biochemistry and physiology of methylotrophic bacteria, which could be extended to obligate methylotrophs and to the comparison of serine cycle versus RuMP- and CBB-utilizing methylotrophs. Methanol is currently regarded as a highly attractive raw material for microbial bioprocesses [56]. Comprehensive, system-level understanding of methylotrophic metabolism is also expected to improve the biotechnological value of methylotrophy, and this knowledge will serve as a sound basis for a rational remodeling of existing biosynthesis routes and for the design of new synthetic pathways [1].

\section{Methods}

\section{Network reconstruction}

The genome-scale (GS) metabolic network of $M$. extorquens AM1 was reconstructed using procedures recommended for the generation of high-quality reconstructions [21]. The detailed procedure is given in Additional file 1 . Briefly, the process of metabolic reconstruction included the following steps:

1. Generating a draft reconstruction. The genome information was extracted from the MicroScope database [57] on December $9^{\text {th }}, 2009$. Genes annotated for metabolic functions were selected and assembled with biochemical information collected from literature. The data were completed using metabolic databases - mainly Metacyc [24], KEGG [22] -. During the reconstruction process, systematic Blast and interrogation of metabolic databases were performed to refine weak - or missing - genome annotation information or new published data. In some cases this process leads to the re-annotation of genes and the publicly available annotation was corrected accordingly.
2. The reconstruction was refined from all genetic, biochemical, and physiological data available for $M$. extorquens and related species, metabolic (Metacyc, KEGG) and transporter (TCDB, http://www.tcdb. org/) databases, and from self-expertise on methylotrophy (Additional file 1). Metabolite information such as the name, molecular formula and metabolic database identifiers of compounds were included in the network, and refined from available metabolomics data for 157 metabolites [35]. Neutral formulas obtained from PubChem http://pubchem.ncbi.nlm. nih.gov/ were used to validate reaction stoichiometry (mass balancing) including proton balance.

3. Generation of a Gene-to-Protein-to-Reaction (GPR) association network. The GPR association was designed to describe explicitly all the relationships between molecular species and functional activities. Specific identifiers were assigned to enzymes, reactions and metabolites.

4. Gap-filling. A draft metabolic map, drawn using the software Cytoscape [58], was used as starting point for the gap filling process. Gaps were identified from stand-alone reactions or metabolites, and from missing connections in essential metabolic processes. Spontaneous reactions, and reactions or transports without associated genes in $M$. extorquens genome, were added according to metabolic (Metacyc, KEGG) and transport (TCDB) databases.

5. Conversion of the reconstruction into computational format. The reconstruction was loaded into the software CellNetAnalyser [25]. The consistency of the reconstructed network was evaluated from in silico investigations (modeling) and from the ability of the network to explain growth on the most studied carbon sources, namely methanol and succinate. Experimental information [26] was used to identify substrate utilization. Exchange reactions - i.e. exchange with environment - were finally added corresponding to known substrates usage and minimal medium composition.

6. Quality assessment. The quality of the reconstructed network was determined according to [21] by assigning a confidence score to each individual reaction, depending on the evidence for the presence of the reaction, with the highest score given to experimentally demonstrated reactions and the lowest score given to gap-filling reactions.

The detailed list of reactions, metabolites, and other network components, and the GPR association network are given in Additional file 2, 3. The computer model of iRP911 written in Systems Biology Markup Language (sbml) is given in the additional file 16 . 


\section{Determination of the chemical composition of cells}

$M$. extorquens AM1 was grown in fed-batch mode in mineral medium containing methanol as sole carbon and energy source, as described in [19]. For cell dry weight (CDW) determination, $30 \mathrm{ml}$ of culture were centrifuged in a $50 \mathrm{ml}$ falcon tube and washed with deionized water and dried to constant weight at $80^{\circ} \mathrm{C}$. Falcon tubes were incubated for several days at $80^{\circ} \mathrm{C}$ prior to use. For other measurements, cells were harvested by centrifugation at $5000 \mathrm{~g}$ during $5 \mathrm{~min}$. Cell pellets were frozen in liquid nitrogen and stored at $-20^{\circ} \mathrm{C}$ until analysis.

\section{i) Lipid content}

whole cell hydrolysis with subsequent acid methylation of fatty acids was carried out as described in [38] with slight modifications. Cells (10-20 mg CDW) were hydrolyzed with $4 \mathrm{ml}$ of $15 \% \mathrm{NaOH}(\mathrm{w} / \mathrm{v})$ in methanol/water $(1: 1, \mathrm{v} / \mathrm{v})$ for $30 \mathrm{~min}$ at $100^{\circ} \mathrm{C}$. Fatty acid methyl esters (FAMEs) were obtained by addition of $8 \mathrm{ml} 6 \mathrm{M} \mathrm{HCl} /$ methanol $(13: 11, \mathrm{v} / \mathrm{v})$ and incubation for $2.5 \mathrm{hrs}$ at $80^{\circ} \mathrm{C}$. An internal fatty acid standard (3 mg C15:0) was added before hydrolysis for quantification purpose. The methylation yield was measured from the addition of a FAME standard (3 mg C19:0 FAME) after the methylation step. FAMEs were extracted with $5 \mathrm{ml}$ hexane/methyl-tertbutyl ether $(1: 1, \mathrm{v} / \mathrm{v})$ and washed with $6 \mathrm{ml} 1 \% \mathrm{NaOH}$ in water $(\mathrm{w} / \mathrm{v})$. Extracted FAMEs were analyzed by gas chromatography - flame ionization detector (GC-FID) (Agilent Technologies 6850 with 7683B Series injector and FID detector) and a HP-5 column, length 30 m, I.D. $0.25 \mathrm{~mm}$, film $0.25 \mu \mathrm{m}$ (Agilent Technologies). Helium was the carrier gas with a column flow of $2.4 \mathrm{ml} / \mathrm{min}$; detector temperature was set to $300^{\circ} \mathrm{C}$, and inlet to $250^{\circ}$ C. A temperature gradient was run from $190^{\circ} \mathrm{C}$ to $260^{\circ} \mathrm{C}$ at $5^{\circ} \mathrm{C}$ per min. A sample volume of $1 \mu \mathrm{l}$ was injected with a spilt ratio of 30 .

\section{ii) Protein content}

total proteins were quantified by the Biuret method [59], using bovine serum albumine $(2 \mathrm{mg} / \mathrm{ml})$ as standard. This method is independent of protein composition [60]. Cells were hydrolyzed in $0.75 \mathrm{ml} 1 \mathrm{~N} \mathrm{NaOH}(1-2 \mathrm{mg} / \mathrm{ml}$ $\mathrm{CDW})$ at $100^{\circ} \mathrm{C}$ for $5 \mathrm{~min}$. After addition of $0.25 \mathrm{ml}$ of $2.5 \% \mathrm{CuSO}_{4}(\mathrm{w} / \mathrm{v})$, samples were centrifuged and absorption was measured at $550 \mathrm{~nm}$. The composition in amino acids of proteins was determined following hydrolysis in $6 \mathrm{M} \mathrm{HCl}$ at $110^{\circ} \mathrm{C}$ for 22 hours under argon samples were dried and derivatized using the AccQ-Tag ${ }^{\mathrm{TM}}$ Ultra derivatization chemistry (Waters Corp., Milford, MA, USA) according to the manufacturer's instruction. Amino acid derivatives were separated by UPLC (Waters Corp., Milford, MA, USA) using the AccQ-Tag ${ }^{\mathrm{TM}}$ Ultra standard hydrolysate conditions. Amino acid derivatives were detected by UV absorbance.

\section{iii) Carbohydrate content}

the carbohydrate content was measured after hydrolysis of the entire cell pellet. A two-step derivatization was used to convert carbohydrates into oxime trimethylsilyl derivates [61], which were analyzed by GC-FID. For glucose and rhamnose quantification, cells (1-3 mg CDW) were directly subjected to $200 \mu \mathrm{l} 2 \mathrm{M} \mathrm{HCl}$ at $80^{\circ} \mathrm{C}$ for 4 hrs or to $4 \mathrm{M} \mathrm{HCl}$ for $16 \mathrm{hrs}$ for glucosamine quantification, respectively; carbohydrates were stable under these conditions. After neutralization, $50 \mu \mathrm{l}$ of $25 \mathrm{mM}$ lactose solution was added as an internal standard. Samples were vacuum-dried and derivatized for $40 \mathrm{~min}$ with $150 \mu \mathrm{l} 0.5 \mathrm{M}$ hydroxylamine. $\mathrm{HCl}$ in pyridine at $80^{\circ} \mathrm{C}$. After addition of $110 \mu \mathrm{l}$ (trimethylsilyl)trifluoroacetamid (BSTFA), samples were incubated for another $20 \mathrm{~min}$. Separation and quantification of the derivatives were performed by GC-FID as described under lipids except that column flow was set to $2.7 \mathrm{ml} / \mathrm{min}$ and temperature gradient was run from $160^{\circ} \mathrm{C}$ to $310^{\circ} \mathrm{C}$ with $7^{\circ} \mathrm{C}$ per min. iv) Polyhydroxybutyrate (PHB) content

The measurement of the PHB content was performed according to $[62,63]$. Cell pellets $(3-4 \mathrm{mg})$ were lyophilized and subjected to acid methanolysis with $3 \% \mathrm{H}_{2} \mathrm{SO}_{4}$ $(\mathrm{v} / \mathrm{v})$ in methanol/chloroform 1:1 (v/v) for $2.5 \mathrm{hrs}$ at $100^{\circ} \mathrm{C}$. Benzoic acid was added as an internal standard prior methanolysis. Methyl-hyroxybutyryl monomers were extracted after addition of water $(20 \% \mathrm{v} / \mathrm{v})$ and vigorous mixing, The organic phase was analyzed by GCFID with a DB-WAX column, length $15 \mathrm{~m}, \mathrm{I} . \mathrm{D} .0 .32$ $\mathrm{mm}$, film $0.5 \mu \mathrm{m}$. Column flow was set to $1.8 \mathrm{ml} / \mathrm{min}$, detector temperature to $270^{\circ} \mathrm{C}$ and inlet temperature to $240^{\circ} \mathrm{C}$. A sample volume of $1 \mu \mathrm{l}$ was injected with a split ratio of 2 . Temperature gradient was run from $90^{\circ}$ $\mathrm{C}$ to $230^{\circ} \mathrm{C}$ at $40^{\circ} \mathrm{C} / \mathrm{min}$.

\section{v) DNA content}

The DNA content was calculated from that in E. coli [64], using appropriate corrections to account for the size of $M$. extorquens genome and for its growth rate on methanol.

\section{vi) RNA content}

The RNA content was determined from the amount of ribose released after acidic hydrolysis $(2 \mathrm{M} \mathrm{HCl}$ for 2 hrs), assuming that all ribose was derived from RNA. The hydrolysis yield was determined from commercial RNA and data were corrected accordingly. Ribose was quantified as described under iii).

\section{vii) Polyamine content}

The polyamine content was calculated from that in $E$. coli. The occurrence of putrescine in methanol-grown M. extorquens cells was also confirmed by GC-MS-MS.

\section{viii) Carotenoid content}

Data were taken from [65]. Based on their experimentally determined chemical properties, they were assumed to be spirilloxanthin-like carotenoids. 


\section{ix) Content in intracellular metabolites}

Data - which included both the nature and amounts of metabolites - were taken from [34,35], [32,33], [66], and [7]. The amounts of Coenzyme A thioesters were determined by P. Kiefer (unpublished data). The content in tetra-aminoptherin and related cofactors were obtained from $[7,66]$. The contents in other cofactors were calculated from that in E.coli [64], using appropriate corrections.

\section{viii) Inorganic ions}

The amounts of inorganic ions were calculated from that in E.coli [64], using appropriate corrections.

The complete details of the biomass composition of $M$. extorquens, as they result from the above investigations or calculations, are given in Additional file 4.

\section{Cultivation and labeling experiment}

Batch cultivations (three biological replicates) of $M$. extorquens AM1 were carried out at $28^{\circ} \mathrm{C}$ in minimal medium, in a bioreactor (Infors-HT, Bottmingen, Switzerland), as described previously [19]. Cultivations carried out for the purpose of steady-state $\left[{ }^{13} \mathrm{C}\right]$-methanol experiments were performed like in [19]. The cultivations were aerated with $5 \%$ natural labeled $\mathrm{CO}_{2}$ to remove the ${ }^{13} \mathrm{CO}_{2}$ produced by the bacteria from $\left[{ }^{13} \mathrm{C}\right]-$ methanol. Under this condition, only $4.6 \pm 0.4 \%$ of total $\mathrm{CO}_{2}$ was found to derive from $\left[{ }^{13} \mathrm{C}\right]$-methanol oxidation.

\section{In silico calculations}

The metabolic network - containing 1139 (m) reactions 977 (n) metabolites - was converted into a mathematical model corresponding to a $\mathrm{m} \times \mathrm{n}$ matrix defining the stoichiometric coefficient of reactions. Calculations of steady-state fluxes were performed using the software CellNetAnlyser [25] and Matlab (Mathworks, Inc.). Flux Balance Analysis (FBA) calculations were performed using various objective functions, as indicated in the text. Despite the genome annotation revealed the occurrence of a potential photosynthetic machinery, all calculations were performed assuming that no photosynthesis operated since no phototrophic behavior was reported for M. extorquens. Calculation of EFMs in the methylotrophic network was performed using the solver EFMTool [67]. They were calculated assuming no maintenance energy. To be compared with experimental data, the obtain biomass yields $(\mathrm{Y})$ of the EFMs were calculated as following:

$$
Y=\frac{\mu_{\max }}{\frac{q_{s i} \times \mu_{\max }}{\mu_{i}}+N_{G A M_{s}}} \times \frac{1000}{M W_{s}}
$$

With $\mu_{\max }$ : theoretical maximum growth rate (calculated to be 0.201 for the methylotrophic network from
FBA simulations); $\mu_{\mathrm{i}}$ : growth rate of the $\mathrm{EFM}_{\mathrm{i}}(=0.1)$; $\mathrm{q}_{\mathrm{si}}$ : substrate uptake rate of the $\mathrm{EFM}_{\mathrm{i}}$, in $\mathrm{mmol} \cdot \mathrm{g}^{-1} \cdot \mathrm{h}^{-}$ ${ }^{1}$; $\mathrm{NGAM}_{\mathrm{s}}$ : corresponding substrate uptake to fulfill non-growth associated maintenance energy, i.e. 1.9 $\mathrm{mmol} \cdot \mathrm{g}^{-1} \cdot \mathrm{h}^{-1} ; \mathrm{MW}_{\mathrm{s}}$ : molecular weight of the substrate. Minimal cut sets [42] were calculated on the calculated EFMs, using biomass production as target function.

\section{${ }^{13} \mathrm{C}$ Metabolic Flux Analysis}

The distribution of metabolic fluxes during methylotrophic growth was determined from ${ }^{13} \mathrm{C}$-labeling data collected during steady-state growth of $M$. extorquens on $\left[{ }^{13} \mathrm{C}\right]$-methanol. The distribution of ${ }^{13} \mathrm{C}$-isotopomers in metabolites can be accurately determined by NMR or MS [45,68,69], alone or in combination [19]. Here the ${ }^{13} \mathrm{C}$-isotopomers of proteinogenic amino-acids were measured by the two methods. NMR spectra were monitored as described in [19] and LC-MS analysis were performed using Rheos 2200 HPLC system (Flux Instruments) coupled to an LTQ Orbitrap mass spectrometer (Thermo Fisher Scientific), equipped with an electrospray ionization probe and the amino acid were separated on a pHILIC column $(150 \times 2.0 \mathrm{~mm}$, particle size $5 \mu \mathrm{m}$; Sequant, Umea, Sweden), following a procedure described [34]. A total of 193 isotopomer data - including 137 NMR data plus 56 MS data - were collected (Additional file 12). The metabolic network considered for flux calculations contained 65 reactions - including 7 reversible reactions - describing $M$. extorquens central metabolism, according to the topology of the methylotrophic network (Additional file 17). Flux calculations were performed using the software 13C-Flux [70], which uses both mass balances and carbon atom transitions to describe the metabolic. The methanol uptake rate and the requirements in biomass precursors, determined from data in Additional file 4, 11, were constrained. The confidence on the measured fluxes was determined using the sensitivity analysis module of 13C-Flux. Results were expressed as absolute fluxes in mmol. $\mathrm{g}^{-1} \cdot \mathrm{h}^{-}$ 1 +/- standard deviations.

\section{Additional material}

Additional file 1: Work flow of the reconstruction and reduction processes. The work flow of the reconstruction was performed similarly to the protocol recommended for the generation of high-quality reconstruction (Thiele \& Palsson, 2010).

Additional file 2: List of reactions of the reconstruction (iRP911)

Additional file 3: List of metabolites of the reconstruction (iRP911)

Additional file 4: Detailed biomass composition

Additional file 5: Table of substrate usage by $M$. extorquens AM1 from experimentally observed phenotype and Flux Balance Analysis using the genome scale network (iRP911). 
Additional file 6: Electron flow through the metabolic network of M. extorquens AM1. The schemas represent the reactions involved in electron flow in $M$. extorquens AM1 like it appear from the network reconstruction (iRP911). Detail on the reaction, given with identifiers of the type R-XXXX, can be found in the Additional file 2

Additional file 7: Methylotrophic network. The reactions of the GS network were included or excluded from the methylotrophic network based on multi-criteria analysis. The list of considered criteria is given here as well as the score for each reaction of the GS network. The list of reactions included in the methylotrophic network are indicated as ' 1 ' in the 'methylotrophic network' column.

Additional file 8: EFM analysis of the primary assimilation pathways and connectivity of biomass precursors. The EFMs were calculated for the conversion of methanol into 13 key carbon precursors. The main properties of the calculated EFMs are given.

Additional file 9: Reaction essentiality in the methylotrophic network. The graph displays the essentiality or dispensability of reactions and showx the experimental evidence for gene essentiality. Reaction essentiality was analyzed using Minimal Cut Set calculation [42] applied to the set of EFMs [30] allowing biomass production from methanol. Fragility Coefficients (FCs) were calculated from the MCSs [42]. Reactions having a FC of 1 were identified as essential (red arrows), and reactions with a FC $<1$ were considered as dispensable (blue arrows). The enzyme (s) catalyzing the network reactions are represented by boxes. The experimental phenotypes of mutants affected for these enzymes are displayed using a color code: red box: lethal phenotype, blue box: nonlethal phenotype, black box: no experimental data available. The accuracy of the model prediction is indicated upper the bar for each class of reaction.

Additional file 10: Table of published mutant phenotypes and associated genes and reactions

Additional file 11: Growth parameters measured for the 3 replicate 13C-methanol labeling experiments

Additional file 12: Fitting of the isotopomers data collected during 13C-methanol labeling experiments

Additional file 13: Flux distributions and sensitivity analysis. Only $\mathrm{C}$ assimilation was considered during flux calculation due to the high difference in range of $\mathrm{C} 1$ dissimilation and assimilation. Measured methanol uptake rate was considered subsequently.

Additional file 14: Quality of isotopomer fitting. Comparison of experimental and collected isotopomer values for the three biological replicates. The isotopomer data include both LC-MS and 2D-NMR (HSQC and TOCSY) data. Flux calculation and fitting were performed using the software 13CFlux (Wiechert et al, 2001). A) Experimental values (+/standard deviation) are plotted against theoretical values. B) Residuum of the calculated data.

Additional file 15: Flux variability in the 3 biological replicates. Comparison of the flux distribution obtained for the three biological replicates. The flux calculation and the sensitivity analysis were performed using the software 13CFLUX (Wiechert et al, 2001). The fluxes were normalized by the flux of entry of the C1-units in central metabolism (SHMT: serine hydroxymethyltransferase). Flux distributions were found to be similar except slight changes through the $\mathrm{C} 3 / \mathrm{C}_{4}$ interconversions (pyruvate kinase (PK), pyruvate dikynase (PPDK), malic enzyme (ME) and the phosphoenolpyruvate carboxykinase (PEPCK)), and through the Entner-Doudorof pathway.

Additional file 16: Computer model written in Systems Biology Markup Language of the genome-scale metabolic network iRP911 Additional file 17: Ftbl file describing the network used for flux calculation

\section{Acknowledgements}

We thank Philipp Christen for cultivation of $M$. extorquens AM1 in bioreactors. We thank Birgit Roth Zgraggen of the Functional Genomic Center Zurich for performing amino acid quantification. This work was supported by ETH Zurich, Research Grant ETH-25 08-2. The Swiss Academy of Engineering Science (SATW) and the Centre Français pour l'Accueil et les Echanges Internationaux (Egide) supported the work with a travel grant (Germaine de Staël program). The work carried out at the LISBP (Toulouse, France) was supported by the Région Midi-Pyrénées, the European Regional Development Fund (ERDF), the French Ministry for Higher Education \& Research, the SICOVAL, and the Réseau RMN Midi-Pyrénées.

\section{Author details}

${ }^{1}$ Institute of Microbiology, ETH Zürich, 8093 Zürich, Switzerland. ${ }^{2}$ Université de Toulouse; INSA, UPS, INP; LISBP, 135 Avenue de Rangueil, F-31077 Toulouse, France. ${ }^{3}$ INRA, UMR792, Ingénierie des Systèmes Biologiques et des Procédés, F-31400 Toulouse, France. ${ }^{4}$ CNRS, UMR5504, F-31400 Toulouse, France.

\section{Authors' contributions}

RP performed the reconstruction and the modeling. KS and PK performed the determination of the biomass composition. RP performed the in silico analysis (EFM, MCS, FBA). RP and SM performed the NMR measurement. RP and PK performed the MS measurement. RP performed the data treatment. $\mathrm{RP}$ performed the metabolic flux analysis and statistical analysis. RP and JCP analyzed the results. RP, JV and JCP wrote the manuscript. RP, JV and JCP conceived the study. All authors read and approved the final manuscript.

Received: 6 August 2011 Accepted: 10 November 2011 Published: 10 November 2011

\section{References}

1. Schrader J, Schilling M, Holtmann D, Sell D, Filho MV, Marx A, Vorholt JA: Methanol-based industrial biotechnology: current status and future perspectives of methylotrophic bacteria. Trends Biotechnol 2009, 27:107-115.

2. Brautaset $T$, Jakobsen $O M$, Josefsen $K D$, Flickinger MC, Ellingsen TE: Bacillus methanolicus: a candidate for industrial production of amino acids from methanol at 50 degrees C. Appl Microbiol Biotechnol 2007, 74:22-34.

3. Chistoserdova L, Kalyuzhnaya MG, Lidstrom ME: The expanding world of methylotrophic metabolism. Annu Rev Microbiol 2009, 63:477-499.

4. Delmotte N, Knief C, Chaffron S, Innerebner G, Roschitzki B, Schlapbach R, von Mering $C$, Vorholt JA: Community proteogenomics reveals insights into the physiology of phyllosphere bacteria. Proc Natl Acad Sci USA 2009, 106:16428-16433.

5. Chistoserdova L: Modularity of methylotrophy, revisited. Environ Microbiol 2011, 13:2603-2622.

6. Van Dien SJ, Lidstrom ME: Stoichiometric model for evaluating the metabolic capabilities of the facultative methylotroph Methylobacterium extorquens AM1, with application to reconstruction of C(3) and C(4) metabolism. Biotechnol Bioeng 2002, 78:296-312.

7. Crowther GJ, Kosaly G, Lidstrom ME: Formate as the Main Branchpoint for Methylotrophic Metabolism in Methylobacterium extorquens AM1. J Bacteriol 2008

8. Van Dien SJ, Strovas T, Lidstrom ME: Quantification of central metabolic fluxes in the facultative methylotroph methylobacterium extorquens AM1 using 13C-label tracing and mass spectrometry. Biotechnol Bioeng 2003, 84:45-55.

9. Corpe WA, Rheem S: Ecology of the methylotrophic bacteria on living leaf surfaces. FEMS Microbiology Letters 1989, 62:243-249.

10. Sy A, Timmers AC, Knief C, Vorholt JA: Methylotrophic metabolism is advantageous for Methylobacterium extorquens during colonization of Medicago truncatula under competitive conditions. Appl Environ Microbiol 2005, 71:7245-7252.

11. Abanda-Nkpwatt D, Musch M, Tschiersch J, Boettner M, Schwab W: Molecular interaction between Methylobacterium extorquens and seedlings: growth promotion, methanol consumption, and localization of the methanol emission site. J Exp Bot 2006, 57:4025-4032

12. Large PJ, Peel D, Quayle JR: Microbial growth on C(1) compounds. 3. Distribution of radioactivity in metabolites of methanol-grown Pseudomonas AM1 after incubation with [C]methanol and [C] bicarbonate. Biochem J 1962, 82:483-488.

13. Large PJ, Peel D, Quayle JR: Microbial growth on C(1) compounds. 4. Carboxylation of phosphoenolpyruvate in methanol-grown Pseudomonas AM1. Biochem J 1962, 85:243-250. 
14. Large PJ, Quayle JR: Microbial growth on C(1) compounds. 5. Enzyme activities in extracts of Pseudomonas AM1. Biochem J 1963, 87:386-396.

15. Chistoserdova L, Vorholt JA, Thauer RK, Lidstrom ME: C1 transfer enzymes and coenzymes linking methylotrophic bacteria and methanogenic Archaea. Science 1998, 281:99-102.

16. Vorholt JA: Cofactor-dependent pathways of formaldehyde oxidation in methylotrophic bacteria. Arch Microbiol 2002, 178:239-249.

17. Skovran E, Crowther GJ, Guo X, Yang S, Lidstrom ME: A systems biology approach uncovers cellular strategies used by Methylobacterium extorquens AM1 during the switch from multi- to single-carbon growth. PLoS One 2010, 5:e14091.

18. Erb TJ, Berg IA, Brecht V, Muller M, Fuchs G, Alber BE: Synthesis of C5dicarboxylic acids from C2-units involving crotonyl-CoA carboxylase/ reductase: the ethylmalonyl-CoA pathway. Proc Natl Acad Sci USA 2007, 104:10631-10636.

19. Peyraud R, Kiefer P, Christen P, Massou S, Portais JC, Vorholt JA: Demonstration of the ethylmalonyl-CoA pathway by using $13 \mathrm{C}$ metabolomics. Proc Natl Acad Sci USA 2009, 106:4846-4851.

20. Vuilleumier S, Chistoserdova L, Lee MC, Bringel F, Lajus A, Zhou Y, Gourion B, Barbe V, Chang J, Cruveiller S, et al: Methylobacterium genome sequences: a reference blueprint to investigate microbial metabolism of C1 compounds from natural and industrial sources. PLoS One 2009, 4: e5584.

21. Thiele I, Palsson BO: A protocol for generating a high-quality genomescale metabolic reconstruction. Nat Protoc 2010, 5:93-121.

22. Kanehisa M, Goto S: KEGG: kyoto encyclopedia of genes and genomes. Nucleic Acids Res 2000, 28:27-30.

23. Saier MH, Tran CV, Barabote RD: TCDB: the Transporter Classification Database for membrane transport protein analyses and information. Nucleic Acids Res 2006, 34:D181-186

24. Caspi R, Altman T, Dale JM, Dreher K, Fulcher CA, Gilham F, Kaipa P, Karthikeyan AS, Kothari A, Krummenacker M, et al: The MetaCyc database of metabolic pathways and enzymes and the BioCyc collection of pathway/genome databases. Nucleic Acids Res 2010, 38:D473-479.

25. Klamt S, Saez-Rodriguez J, Gilles ED: Structural and functional analysis of cellular networks with CellNetAnalyzer. BMC Syst Biol 2007, 1:2.

26. Knief C, Frances L, Vorholt JA: Competitiveness of diverse Methylobacterium strains in the phyllosphere of Arabidopsis thaliana and identification of representative models, including $M$. extorquens PA1. Microb Ecol 2010, 60:440-452.

27. Southall SM, Doel JJ, Richardson DJ, Oubrie A: Soluble aldose sugar dehydrogenase from Escherichia coli: a highly exposed active site conferring broad substrate specificity. J Biol Chem 2006, 281:30650-30659.

28. Van Dien SJ, Okubo Y, Hough MT, Korotkova N, Taitano T, Lidstrom ME: Reconstruction of C(3) and C(4) metabolism in Methylobacterium extorquens AM1 using transposon mutagenesis. Microbiology 2003, 149:601-609.

29. Portais JC, Delort AM: Carbohydrate cycling in micro-organisms: what can (13)C-NMR tell us? FEMS Microbiol Rev 2002, 26:375-402.

30. Schuster S, Dandekar T, Fell DA: Detection of elementary flux modes in biochemical networks: a promising tool for pathway analysis and metabolic engineering. Trends Biotechnol 1999, 17:53-60.

31. Bosch G, Skovran E, Xia Q, Wang T, Taub F, Miller JA, Lidstrom ME, Hackett M: Comprehensive proteomics of Methylobacterium extorquens AM1 metabolism under single carbon and nonmethylotrophic conditions. Proteomics 2008, 8:3494-3505.

32. Guo X, Lidstrom ME: Physiological analysis of Methylobacterium extorquens AM1 grown in continuous and batch cultures. Arch Microbiol 2006, 186:139-149.

33. Guo X, Lidstrom ME: Metabolite profiling analysis of Methylobacterium extorquens AM1 by comprehensive two-dimensional gas chromatography coupled with time-of-flight mass spectrometry. Biotechnol Bioeng 2008, 99:929-940.

34. Kiefer P, Portais JC, Vorholt JA: Quantitative metabolome analysis using liquid chromatography-high-resolution mass spectrometry. Anal Biochem 2008, 382:94-100.

35. Kiefer P, Delmotte N, Vorholt JA: Nanoscale lon-Pair Reversed-Phase HPLC-MS for Sensitive Metabolome Analysis. Anal Chem 2010.

36. Lee MC, Chou HH, Marx CJ: Asymmetric, bimodal trade-offs during adaptation of Methylobacterium to distinct growth substrates. Evolution 2009, 63:2816-2830.
37. Schmidt S, Christen P, Kiefer P, Vorholt JA: Functional investigation of methanol dehydrogenase-like protein XoxF in Methylobacterium extorquens AM1. Microbiology 2010, 156:2575-2586.

38. Sasser M: Identification of bacteria through fatty acid analysis. In Methods in Phytobacteriology. Edited by: Klement Z, Rudolph K, Sands DC. Budapest: Akademiai Kiado; 1990:199-204.

39. Okubo Y, Yang S, Chistoserdova L, Lidstrom ME: Alternative route for glyoxylate consumption during growth on two-carbon compounds by Methylobacterium extorquens AM1. J Bacteriol 2010, 192:1813-1823.

40. Erb TJ, Frerichs-Revermann L, Fuchs G, Alber BE: The apparent malate synthase activity of Rhodobacter sphaeroides is due to two paralogous enzymes, (3S)-Malyl-coenzyme A (CoA)/\{beta\}-methylmalyl-CoA lyase and (3S)- Malyl-CoA thioesterase. J Bacteriol 2010, 192:1249-1258.

41. Hagemeier $\mathrm{CH}$, Chistoserdova L, Lidstrom ME, Thauer RK, Vorholt JA Characterization of a second methylene tetrahydromethanopterin dehydrogenase from Methylobacterium extorquens AM1. Eur J Biochem 2000, 267:3762-3769

42. Klamt S, Gilles ED: Minimal cut sets in biochemical reaction networks. Bioinformatics 2004, 20:226-234.

43. Chistoserdova L, Chen SW, Lapidus A, Lidstrom ME: Methylotrophy in Methylobacterium extorquens AM1 from a genomic point of view. J Bacteriol 2003, 185:2980-2987.

44. Edwards JS, Palsson BO: Robustness analysis of the Escherichia coli metabolic network. Biotechnol Prog 2000, 16:927-939.

45. Massou S, Nicolas C, Letisse F, Portais JC: NMR-based fluxomics: quantitative 2D NMR methods for isotopomers analysis. Phytochemistry 2007, 68:2330-2340.

46. Large PJ, Peel D, Quayle JR: Microbial growth on C1 compounds. II. Synthesis of cell constituents by methanol- and formate-grown Pseudomonas AM 1, and methanol-grown Hyphomicrobium vulgare. Biochem J 1961, 81:470-480.

47. Hacking AJ, Quayle JR: Purification and properties of malyl-coenzyme A lyase from Pseudomonas AM1. Biochem J 1974, 139:399-405.

48. Smejkalova H, Erb TJ, Fuchs G: Methanol assimilation in Methylobacterium extorquens AM1: demonstration of all enzymes and their regulation. PLoS One 2010, 5.

49. Mahadevan R, Palsson BO, Lovley DR: In situ to in silico and back: elucidating the physiology and ecology of Geobacter spp. using genome-scale modelling. Nat Rev Microbiol 2011, 9:39-50.

50. Mahadevan R, Lovley DR: The degree of redundancy in metabolic genes is linked to mode of metabolism. Biophys J 2008, 94:1216-1220.

51. Quayle JR, Pfennig N: Utilization of methanol by rhodospirillaceae. Arch Microbiol 1975, 102:193-198.

52. McKinlay JB, Harwood CS: Carbon dioxide fixation as a central redox cofactor recycling mechanism in bacteria. Proc Natl Acad Sci USA 2010, 107:11669-11675

53. Laguna R, Tabita FR, Alber BE: Acetate-dependent photoheterotrophic growth and the differential requirement for the Calvin-Benson-Bassham reductive pentose phosphate cycle in Rhodobacter sphaeroides and Rhodopseudomonas palustris. Arch Microbiol 2010.

54. Fall $R$, Benson AA: Leaf methanol - The simplest natural product from plants. Trends in Plant Science 1996, 1:296-301.

55. Huve K, Christ MM, Kleist E, Uerlings R, Niinemets U, Walter A, Wildt J: Simultaneous growth and emission measurements demonstrate an interactive control of methanol release by leaf expansion and stomata. J Exp Bot 2007, 58:1783-1793.

56. Olah GA, Goeppert A, Prakash GKS: Beyond oil and gas: the methanol economy, Second, Updated and Enlarged Edition Wiley-VCH; 2009.

57. Vallenet D, Engelen S, Mornico D, Cruveiller S, Fleury L, Lajus A, Rouy Z, Roche D, Salvignol G, Scarpelli C, Medigue C: MicroScope: a platform for microbial genome annotation and comparative genomics. Database (Oxford) 2009, 2009:bap021

58. Shannon P, Markiel A, Ozier O, Baliga NS, Wang JT, Ramage D, Amin N, Schwikowski B, Ideker T: Cytoscape: a software environment for integrated models of biomolecular interaction networks. Genome Res 2003, 13:2498-2504.

59. Herbert DP, P J, Strange RE: Chemical analysis of microbial cells. In Methods in Microbiology. Volume 5B. Edited by: Norris JR, D W. London and New York: Academic Press; 1971:209-344.

60. Sapan CV, Lundblad RL, Price NC: Colorimetric protein assay techniques. Biotechnology and Applied Biochemistry 1999, 29:99-108. 
61. Kiefer $P$, Heinzle $E$, Wittmann $C$ : Influence of glucose, fructose and sucrose as carbon sources on kinetics and stoichiometry of lysine production by Corynebacterium glutamicum. J Ind Microbiol Biotechnol 2002, 28:338-343.

62. Braunegg G, Sonnleitner B, Lafferty RM: Rapid Gas-Chromatographic Method for Determination of Poly-Beta-Hydroxybutyric Acid in Microbial Biomass. European Journal of Applied Microbiology and Biotechnology 1978, 6:29-37.

63. Jan S, Roblot C, Goethals G, Courtois J, Courtois B, Saucedo JEN, Seguin JP, Barbotin JN: Study of Parameters Affecting Poly-3-Hydroxybutyrate Quantification by Gas-Chromatography. Analytical Biochemistry 1995, 225:258-263.

64. Neidhardt FC: Chemical composition of Escherichia coli. In Escherichia coli and Salmonella: Cellular and Molecular Biology. Volume 1. Edited by: Neidhardt FC, Curtiss R, Ingraham JL, Lin ECC, Low KB, Magasanik B, et al. Washington, D.C.: American Society for Microbiology Press; 1996:3-6.

65. Konovalova HM, Shylin SO, Rokytko PV: Characteristics of carotinoids of methylotrophic bacteria of Methylobacterium genus. Mikrobiol Z 2007 69:35-41.

66. Vorholt JA, Chistoserdova L, Lidstrom ME, Thauer RK: The NADP-dependent methylene tetrahydromethanopterin dehydrogenase in Methylobacterium extorquens AM1. J Bacteriol 1998, 180:5351-5356.

67. Terzer M, Stelling J: Large-scale computation of elementary flux modes with bit pattern trees. Bioinformatics 2008, 24:2229-2235.

68. Massou S, Nicolas C, Letisse F, Portais JC: Application of 2D-TOCSY NMR to the measurement of specific(13C-enrichments in complex mixtures of 13C-labeled metabolites. Metab Eng 2007, 9:252-257.

69. Kiefer P, Nicolas C, Letisse F, Portais JC: Determination of carbon labeling distribution of intracellular metabolites from single fragment ions by ion chromatography tandem mass spectrometry. Anal Biochem 2007, 360:182-188.

70. Wiechert W, Mollney M, Petersen S, de Graaf AA: A universal framework for $13 \mathrm{C}$ metabolic flux analysis. Metab Eng 2001, 3:265-283.

doi:10.1186/1752-0509-5-189

Cite this article as: Peyraud et al:: Genome-scale reconstruction and system level investigation of the metabolic network of Methylobacterium extorquens AM1. BMC Systems Biology 2011 5:189.

\section{Submit your next manuscript to BioMed Central and take full advantage of:}

- Convenient online submission

- Thorough peer review

- No space constraints or color figure charges

- Immediate publication on acceptance

- Inclusion in PubMed, CAS, Scopus and Google Scholar

- Research which is freely available for redistribution

Submit your manuscript at www.biomedcentral com/submit 